\title{
Electron Transfer in Gaseous Positively Charged Peptides - Relation to Mass Spectrometry
}

\author{
Jack Simons
}

Contents

1. Introduction 164

1.1 The electron-capture event involves electron transfer $\quad 165$

1.2 Intra-peptide electron transfer can also occur 166

2. The Theoretical Challenges and Examples of How the Studies are Performed

2.1 Theoretical considerations 170

2.2 Illustrative examples 173

3. Relation to More Common Forms of Electron Transfer 178

Acknowledgment 182

References 182

\begin{abstract}
Special theoretical tools are needed to carry out $a b$ initio simulations of (i) electron transfer from a negatively charged donor (i.e., an anion donor) to a positively charged polypeptide and (ii) electron transfer within such a peptide from Rydberg orbitals on positive sites (e.g., protonated amines on side chains) to disulfide or amide bond sites. Basis sets capable of describing several Rydberg states as well as states with an electron attached to an SS $\sigma^{\star}$ or OCN $\pi^{\star}$ orbital must be used. Electron correlation is important to include for some states, and methods that allow one to obtain excited states of the same spin and spatial symmetry must be employed. Tools for treating surface hopping between states are also crucial. Examples of applying such tools to anion-to-peptide and intra-peptide electron-transfer processes are presented. It is demonstrated that intra-peptide electron transfer from Rydberg orbitals can occur over long distances $(15 \AA)$ and can take place in
\end{abstract}

Chemistry Department and Henry Eyring Center for Theoretical Chemistry, University of Utah, Salt Lake City, UT 84112, USA 
both through-space and through-bond paths. Similarities and differences with other electron-transfer processes in chemistry are also discussed.

Keywords: electron-capture dissociation; electron-transfer dissociation; electron transfer; Rydberg orbital; Landau-Zener theory

\section{INTRODUCTION}

Electron-capture dissociation (ECD) [1] and electron-transfer dissociation (ETD) [2] mass spectroscopic methods have shown much utility and promise for sequencing peptides and proteins. A strong point of both techniques is their propensity for selectively cleaving disulfide and $\mathrm{N}-\mathrm{C}_{\alpha}$ bonds and for doing so over a wide range of the backbone, thus producing many different fragment ions, unlike collision-induced dissociation (CID) or infrared multiphoton dissociation (IRMPD). ECD and ETD also preserve labile sidechains with posttranslational modifications. Parallel with many advances in the experimental development and improvement of these methods, theoretical studies have been carried out by several groups to try to determine the mechanism(s) [3] by which electron attachment leads to these specific bond cleavages as well as how the initial electron attachment occurs.

In both ECD and ETD experimental approaches, a positively charged sample of a polypeptide enters the gas phase (usually via electrospray), after which ions of specific mass to charge ratio are selected. Usually, the positive charging is induced by subjecting the solution-phase sample to acidic conditions prior to electrospray. An example of a relatively simple polypeptide is shown in Figure 1 as a means for introducing several concepts and terminology.

In ETD, an anion donor collides with the positively charged peptide and transfers an electron to the peptide; subsequent to this intermolecular electron transfer, the peptide undergoes cleavage at one of its $\mathrm{N}-\mathrm{C}_{\alpha}$ or $\mathrm{S}-\mathrm{S}$ bonds to form fragment ions. The mass to charge ratios and intensities of the fragment ions are the raw data that is then used to infer the primary sequence of the original

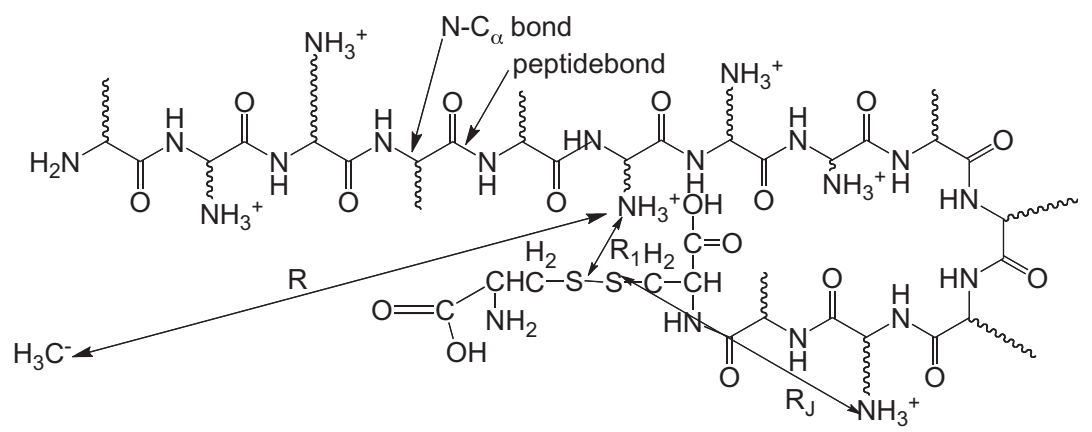

Figure 1 Prototypical polypeptide showing disulfide (SS) linkage, one of many $\mathrm{N}-\mathrm{C}_{\alpha}$ bonds, amino acid side chains (wavy lines), protonated amines on side chains (wavy lines), and one of many peptide bonds. Also shown is an anion donor $\left(\mathrm{H}_{3} \mathrm{C}^{-}\right)$colliding with the peptide. 
polypeptide. In ECD, a free electron (usually having low kinetic energy) rather than a molecular anion collides with the parent polypeptide. This electron is captured and subsequently the peptide undergoes cleavage at one of its $\mathrm{N}-\mathrm{C}_{\alpha}$ or $\mathrm{S}-\mathrm{S}$ bonds. The kind of fragment ions produced (i.e., those arising from $\mathrm{N}-\mathrm{C}_{\alpha}$ or S-S bond cleavage) and their intensities are found to be very similar for ETD and ECD, suggesting that the two processes proceed along very similar mechanistic paths. The detailed mechanism(s) by which the electron attaches to the peptide, where it attaches, and how the $\mathrm{N}-\mathrm{C}_{\alpha}$ or $\mathrm{S}-\mathrm{S}$ bond cleavage then takes place have been the main focuses of our research in this area.

\subsection{The electron-capture event involves electron transfer}

In both ECD and ETD, the initial conditions appropriate to the experiments do not correspond to the ground electronic state of the electron/peptide (ECD) or anion/ peptide (ETD) system. In both cases, there are a myriad of lower-energy electronic states, and this fact presents major challenges to the theoretical study of these processes. In Figure 2, we show qualitative plots of energies as functions of the distance $R$ between a $\mathrm{H}_{3} \mathrm{C}^{-}$anion donor and a polypeptide having total charge $Z$.

The families of electronic states that must be considered in such a study and that are depicted in Figure 2 include:

1. The ion-pair state in which the "excess" electron resides on the donor anion; this state's energy varies strongly with $R$ reflecting the strong Coulomb attraction between the anion donor and the positively charged polypeptide. In Figure 2, this state is shown as rapidly descending as $R$ decreases approximately as expected based on the Coulomb attraction between the anion donor and the peptide of charge $\mathrm{Z}:-14.4 \mathrm{Z} / R$ is in $\mathrm{eV}$, when $R$ is in $\AA$.

2. Families of Rydberg states in which the excess electron has moved from the anion donor to reside in a Rydberg orbital (ground 3s, or excited 3p, 3d, 4s, etc.) on one of the polypeptide's protonated amine side chains. These curves (at least

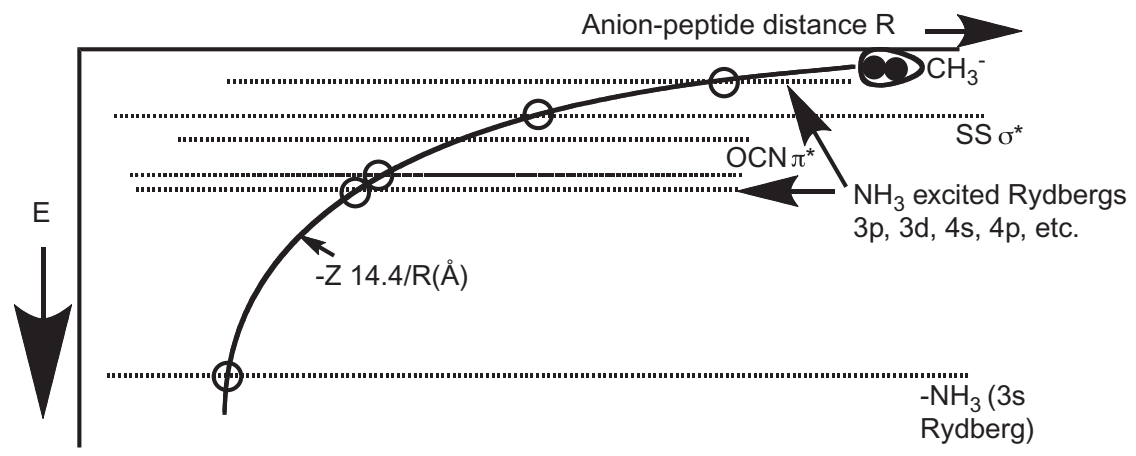

Figure 2 Qualitative plots of the electronic energy surfaces as functions of the anion-topeptide distance $R$, for the anion-peptide collision complex, and for states in which the electron has been transferred from the anion to Rydberg states on one of the peptide's protonated amines, to an SS $\sigma^{*}$ orbital, or to an amide $\pi^{*}$ orbital. 
at long anion-peptide distances) are found to vary rather weakly with $R$ because the anion donor has been rendered neutral, so only charge-dipole and charge-induced-dipole potentials between the peptide and the $\mathrm{H}_{3} \mathrm{C}$ radical exist.

3. One or more states in which the excess electron has moved to reside in an antibonding SS $\sigma^{*}$ orbital of one of the peptide's disulfide linkages.

4. One or more states in which the excess electron has moved to reside in an antibonding OCN $\pi^{*}$ orbital of one of the peptide's amide linkages. The curves of these $\sigma^{*}$ and $\pi^{*}$ vary rather weakly with $R$ for the same reasons as noted above.

Near where we depict the energy surfaces crossing in Figure 2, the pairs of surfaces actually undergo avoided crossings at which they experience a minimum energy splitting that we denote $2 \mathrm{H}_{1,2}$. Moving through each such avoided crossing, the nature of the two states changes. For example, when the ion-pair state approaches the $-\mathrm{NH}_{3} 3$ s ground-Rydberg state from above at the left-most circle in Figure 2, the lower-energy surface corresponds to having the extra electron in the 3s Rydberg orbital; the upper surface has this electron in the methyl lone pair orbital. In contrast, to the left of the circle, the lower surface corresponds to the ion-pair state, while the upper surface is the 3s Rydbergattached state. The evolution of the two states' energies and wave functions through such avoided crossings describes how the interspecies electron transfer occurs. This is the first category of electron-transfer processes one needs to study when investigating ETD or ECD.

In probing ETD experiments, one must be able to characterize the above four families of electronic energy surfaces, and one must have a means of extracting the couplings $\mathrm{H}_{1,2}$ between these states as they undergo avoided crossings. In the studies that our group has undertaken [3h-3w], we have used Landau-Zener (LZ) theory to estimate the probabilities $P$ for an electron being transferred from an anion donor to a Rydberg orbital, an SS $\sigma^{*}$ orbital, or an amide $\pi^{*}$ orbital during a collision beginning on the attractive ion-pair surface that undergoes a crossing with one of the other surfaces. In LZ theory, this probability is computed as

$$
P=1-\exp \left[-\frac{2 \pi \mathrm{H}_{1,2}^{2}}{\hbar v|\Delta F|}\right] \approx \frac{2 \pi \mathrm{H}_{1,2}^{2}}{\hbar v|\Delta F|}
$$

$\mathrm{H}_{1,2}$ is one half the splitting observed when the two energy surfaces undergo their avoided crossing, $v$ the speed at which the tion pair moves through the avoided crossing region, and $\Delta F$ the difference in the slopes of the two energy surfaces as they approach the avoided crossing.

\subsection{Intra-peptide electron transfer can also occur}

Once an electron is transferred to or captured by the polypeptide, various things can happen:

1. If the electron attaches directly to an SS $\sigma^{*}$ orbital, the disulfide bond promptly cleaves [3j] because the $\sigma^{2} \sigma^{* 1}$ electron-attached state is strongly 
repulsive along the S-S bond. This is one path by which disulfide cleavage occurs.

2. If the electron enters an $\mathrm{OCN} \pi^{*}$ orbital, an ${ }^{-} \mathrm{O}-\mathrm{C} \cdot-\mathrm{NH}-\mathrm{C}_{\alpha}$ radical anion center is formed, after which the neighboring $\mathrm{N}-\mathrm{C}_{\alpha}$ bond is weakened and can be cleaved (to produce ${ }^{-} \mathrm{O}-\mathrm{C}=\mathrm{NH}+\cdot{ }^{-} \mathrm{C}_{\alpha}$ ) thus producing the $\mathrm{N}-\mathrm{C}_{\alpha}$ bondcleavage products $[3 \mathrm{~m}]$.

3. If the electron enters a Rydberg orbital on one of the protonated amine sites, in addition to undergoing a cascade of radiative or non-radiative relaxation steps to lower-energy Rydberg states, it can subsequently undergo intra-peptide electron transfer to either an SS $\sigma^{*}$ or an OCN $\pi^{*}$ orbital after which disulfide or $\mathrm{N}-\mathrm{C}_{\alpha}$ bond cleavage can occur $[3 \mathrm{r}, 3 \mathrm{u}-3 \mathrm{w}]$.

For the intra-peptide electron migration to be effective in cleaving an S-S or $\mathrm{N}-\mathrm{C}_{\alpha}$ bond, it must occur before the Rydberg species from which the electron is transferred can decay by some other mechanism. It is believed that electron attachment (in ECD or ETD) at a positively charged side chain initially occurs into an excited-Rydberg orbital after which a decay cascade eventually leads to formation of the ground-Rydberg species. It is known that excited-Rydberg states belonging to protonated or fixed-charge amine sites undergo radiationless relaxation to the ground-Rydberg state in a few to several microseconds. Moreover, we know that the excited-Rydberg states do not undergo $\mathrm{N}-\mathrm{H}$ or $\mathrm{N}-\mathrm{C}$ bond cleavage, but the ground-Rydberg states do (in $c a .10^{-12} \mathrm{~s}$ ). Hence, the intra-peptide electron transfer must occur within a few microseconds of the time the electron attaches to an excited-Rydberg orbital; otherwise, it will relax to the groundRydberg state and $\mathrm{N}-\mathrm{H}$ or $\mathrm{N}-\mathrm{C}$ bond cleavage will occur (ejecting an $\mathrm{H}$ atom or an alkyl radical) terminating the electron's chance to undergo further transfer.

This transfer from a Rydberg orbital to an SS or OCN antibonding orbital is the second family of electron-transfer events that must be considered when studying ECD or ETD. These transfers can occur either through-space or through-bond. To appreciate which Rydberg states are most likely to be involved, qualitative depictions of the energies of states in which the extra electron occupies a Rydberg orbital or an SS $\sigma^{*}$ orbital are shown in Figure 3 as functions of the S-S bond length.

The energy profile of the SS $\sigma^{*}$-attached state is largely repulsive, ${ }^{1}$ but its location, relative to the parent and Rydberg-attached states, depends upon the distance $R$ between the SS bond and the positively charged site whose Coulomb potential acts to move the SS $\sigma^{*}$-attached state up and down in energy as $R$ varies. For example, if $R$ is very large, the energy of the SS $\sigma^{*}$-attached state will be little affected by the stabilizing Coulomb potential of the $-\mathrm{NH}_{3}^{+}$site and thus its energy profile will be as shown by the upper curve in Figure 3. Alternatively, if the $-\mathrm{NH}_{3}^{+}$site is closer to the SS bond, the energy profile will be shifted downward as in the lower curve in Figure 3.

For each instantaneous value of the Coulomb potential experienced by the SS $\sigma^{*}$ orbital, a different Rydberg state will intersect the energy profile of the

${ }^{1}$ This state's energy is weakly attractive at large distances because of van der Waals and charge-induced dipole interactions, but its valence-range character is repulsive. 


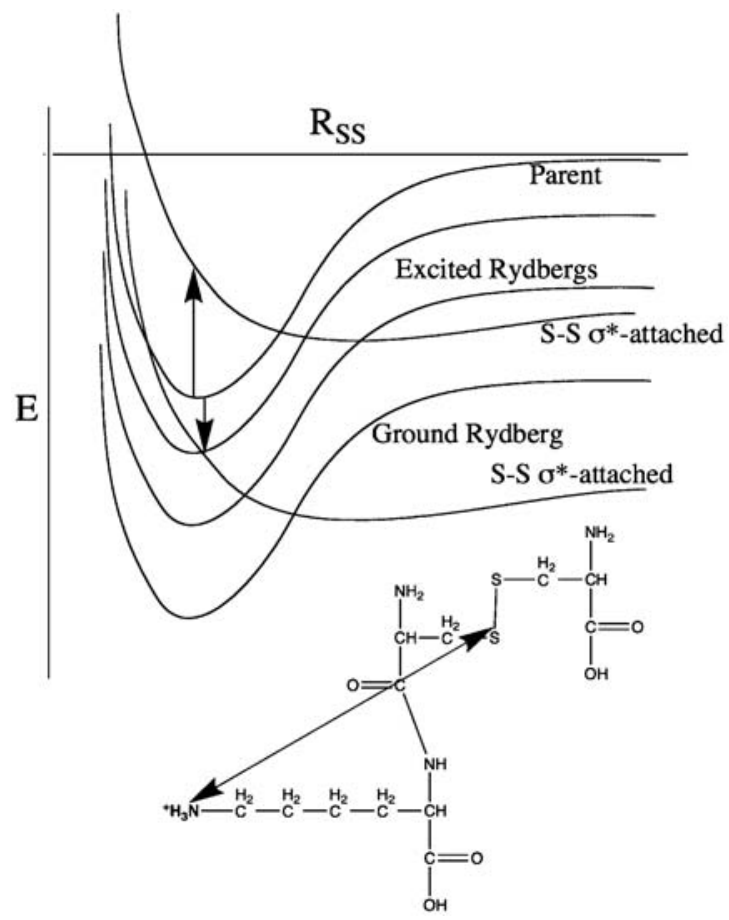

Figure 3 Energies, as functions of the $\mathrm{S}-\mathrm{S}$ bond length, of the parent charged polypeptide (top), of ground and excited-Rydberg states localized on the protonated amine side chain, and of the SS $\sigma^{*}$-attached state in the absence of (upper curve) and in the presence of (lower curve) Coulomb stabilization (appears as Figure 1 in ref. 3s).

SS $\sigma^{*}$-attached state at or near the equilibrium SS bond length $R_{\mathrm{e}}$. In polypeptides containing multiple positively charged sites such as that shown in Figure 4 , the total Coulomb potential $C$

$$
C=-14.4 \sum_{J} \frac{1}{R_{J}}
$$

will determine the energy-placement of the SS $\sigma^{*}$-attached state $\left(R_{J}\right.$ is the distance of the Jth charged site to the SS bond).

Because ETD and ECD experiments are carried out at or near room temperature, the SS and $\mathrm{N}-\mathrm{C}_{\alpha}$ bonds are expected to sample only distances close to their equilibrium values $R_{\mathrm{e}}$. Hence, we focus primarily on the Rydberg states having energies close to that of the SS $\sigma^{*}$-attached or OCN $\pi^{*}$-attached state near $R_{\mathrm{e}}$ when considering intra-peptide electron transfer. In Figure 3, this would be the highest Rydberg state shown.

In the studies our group has undertaken $[3 h-3 w]$ to date, we used LZ theory to estimate the probabilities $P$ for an electron being transferred from such a Rydberg orbital to an SS $\sigma^{*}$ or amide $\pi^{*}$ orbital. In Figure 5 we show actual data from such a study on the $\mathrm{H}_{3} \mathrm{C}-\mathrm{S}-\mathrm{S}-\left(\mathrm{CH}_{2}\right)_{3}-\mathrm{NH}_{3}^{+}$model compound. 


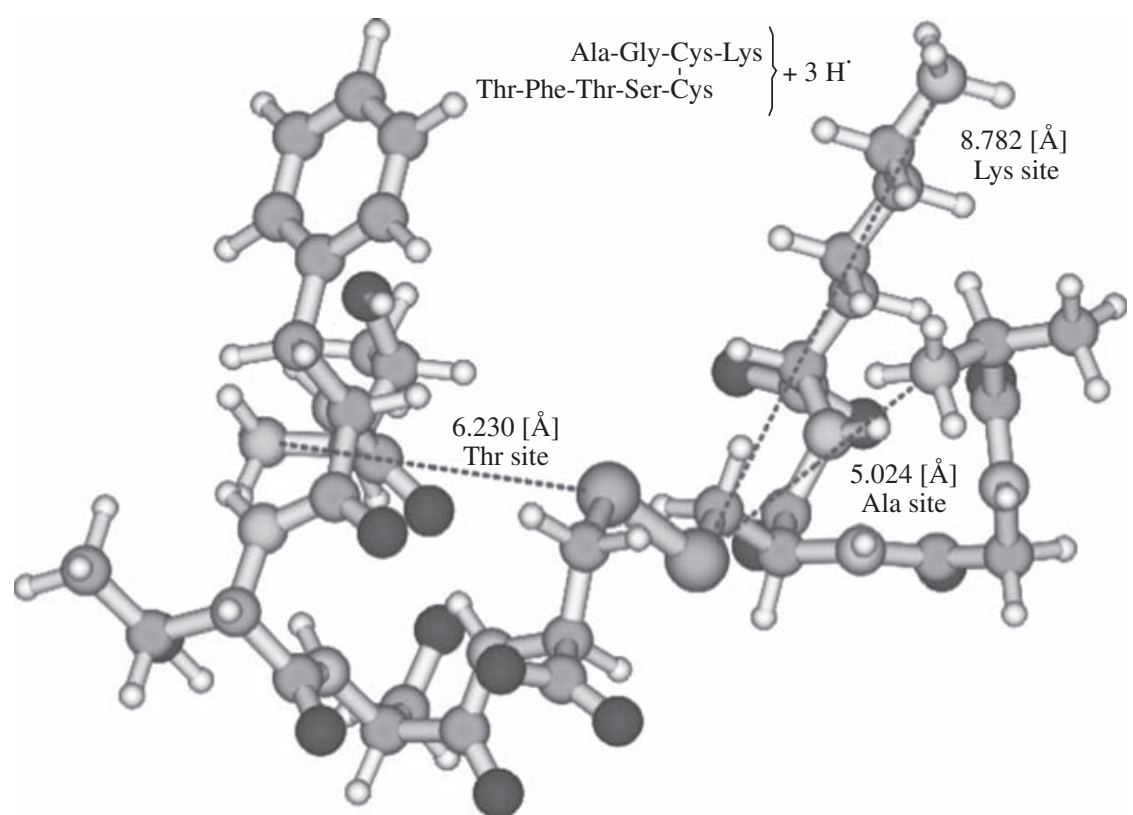

Figure 4 Triply protonated polypeptide containing one SS linkage with the distances $R$, to each positive site labeled by dotted lines (appears in Figure 7 of ref. 3s).

From the data shown in Figure 5, we concluded that it is the excited-Rydberg state that crosses the repulsive SS $\sigma^{*}$-attached state near $R_{\mathrm{e}}$, so this is the state from which electron transfer is most likely to occur. The $82 \mathrm{~cm}^{-1}$ energy value shown in Figure 5 is the electronic coupling matrix element $\mathrm{H}_{1,2}$ connecting the excited-Rydberg and SS $\sigma^{*}$ states, which plays a central role in determining the LZ-estimated probability $P$ of electron transfer (see Equation (1)). In these cases, the rates of electron transfer are computed by multiplying the frequency $v$ at which the S-S bond moves through the curve crossing (we take this to be the harmonic frequency of the SS bond) by the LZ probability $P$. In the LZ formula, the speed $v$ at which the system passes through the crossing region is computed in terms of the speed of the SS vibrational motion.

To illustrate, it was shown in ref. $3 \mathrm{q}$ that $\mathrm{H}_{1,2}$ values in the $300 \mathrm{~cm}^{-1}$ range produce LZ probabilities of $c a$. $0.1-0.5$ for this system. Thus, we can estimate the rates of electron transfer by multiplying the S-S vibrational frequency $v_{\mathrm{SS}}$ ( $c a$. $1.5 \times 10^{13} \mathrm{~s}^{-1}$ ) by the surface hopping probability $(0.1-0.5)$ and then scaling by the ratio of the square of $\left(\mathrm{H}_{1,2} / 300\right)$ :

$$
\text { Rate } \approx(1.5 \text { to } 7.5) \times 10^{12}\left[\frac{\mathrm{H}_{1,2}}{300}\right]^{2} \mathrm{~s}^{-1}
$$

Such estimates allowed us to conclude that the smallest $\mathrm{H}_{1,2}$ that could produce S-S bond cleavage competitive with relaxation from one Rydberg state to another (taking place at $c a .10^{6} \mathrm{~s}^{-1}$ ) should be $\mathrm{H}_{1,2}^{\min } \approx 0.11-0.24 \mathrm{~cm}^{-1}$. Most of the $\mathrm{H}_{1,2}$ values we obtained in our studies to date are substantially larger, 


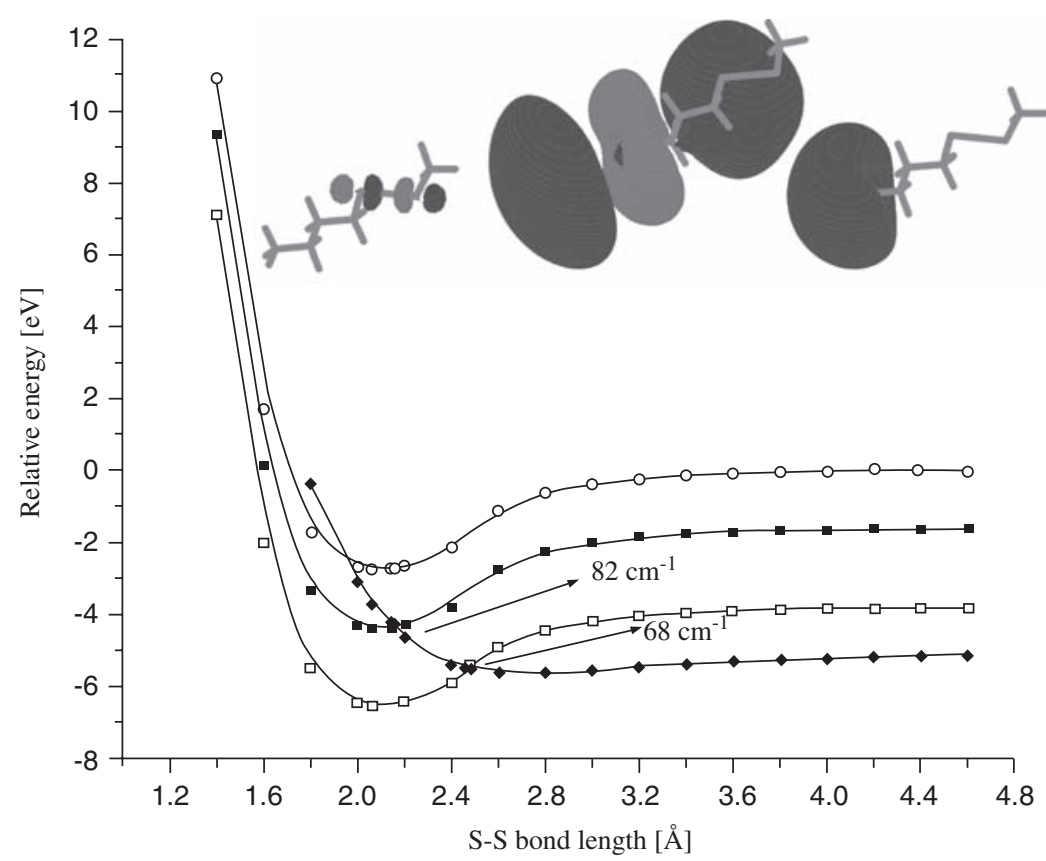

Figure 5 Energies of the parent $\mathrm{H}_{3} \mathrm{C}-\mathrm{S}-\mathrm{S}-\left(\mathrm{CH}_{2}\right)_{3}-\mathrm{NH}_{3}^{+}$cation (open circles), ground Rydberg-attached (open squares), excited Rydberg-attached (filled squares), and $\mathrm{S}-\mathrm{S} \sigma^{*}$-attached (filed diamonds) states as functions of the S-S bond length. Also shown are the SS $\sigma^{*}$ (left), excited-Rydberg (center), and ground-Rydberg (right) orbitals (appears as Figure 4 in ref. 3s).

suggesting that intra-peptide electron transfer can be an important contributor to electrons attaching to and cleaving SS and $\mathrm{N}-\mathrm{C}_{\alpha}$ bonds.

In summary, ETD and ECD processes involve two kinds of electron-transfer events. The first occurs in the initial capture of an electron by the positively charged polypeptide. The second involves intra-peptide electron transfer from a Rydberg orbital residing on a positively charged site to an SS or OCN bond site.

\section{THE THEORETICAL CHALLENGES AND EXAMPLES OF HOW THE STUDIES ARE PERFORMED}

\subsection{Theoretical considerations}

Before discussing specific examples as a tool for illustrating how one uses theory to carry out such studies, we overview a few components of all theoretical investigations of the electron-transfer events we have studied. Specifically, one must be sure to address all of the following issues:

1. Atomic orbital basis sets containing diffuse functions must be used at least for the atoms onto which the electron will attach. This means the sulfur atoms if one is studying disulfide cleavage and the $\mathrm{O}, \mathrm{C}$, and $\mathrm{N}$ atoms (at the site of cleavage) if one is studying $\mathrm{N}-\mathrm{C}_{\alpha}$ cleavage. It is important to then check to 
make sure one obtains a reasonably accurate electron binding energy for the fragment that holds the excess electron upon bond cleavage. For SS bond cleavage, this means verifying that the ${ }^{-} \mathrm{S}-\mathrm{R}$ anion has an electron binding energy near $1.4 \mathrm{eV}$. This is important because the relative energies of the bondattached and Rydberg-attached states determine which Rydberg state is likely to couple to the bond-attached state.

2. The positively charged site to which an electron is to attach must have special basis functions [4-6] attached to it to describe the Rydberg orbitals. This is important because one needs to accurately describe the energies of the Rydberg states in relation to bond-attached states and the Rydberg orbitals' radial extent must be properly represented. To appreciate the sizes of such orbitals, we show in Figure 6 the lowest (labeled 3s, 3p, 3d, 4s, 4p, and $5 s$ because $\mathrm{NH}_{4}^{+}$is isoelectronic with $\mathrm{Na}^{+}$) Rydberg orbitals of $\mathrm{NH}_{4}$.

In each orbital, the outer surface in the figure contains only $60 \%$ of the electron density (i.e., $40 \%$ of the density lies farther from the cation center). Moreover, for each orbital, one can notice the size of the van der Waals surface of the underlying $\mathrm{NH}_{4}^{+}$cation to gain perspective about how large these Rydberg orbitals are. Realizing that the $\mathrm{N}-\mathrm{H}$ bond length is $c a .1 \AA$, it is easy to appreciate that these Rydberg orbitals span (even at the $60 \%$ contour level) $10 \AA$ or more. ${ }^{2}$

3. The theoretical methods used must be capable of describing not only ground but also (several) excited states, including state of the same spatial and spin symmetry. We have found it possible to converge Hartree-Fock self-consistent field (HF-SCF) calculations on excited states by starting the SCF process with a spin-orbital occupancy that describes the desired electronic state. After converging the SCF calculation and checking to make sure it has converged to the correct state, we have employed Møller-Plesset perturbation theory at second order (MP2) to evaluate the energy of each state. A correlated treatment is not so important for the Rydberg-attached states because they

${ }^{2}$ Hydrogenic and Rydberg orbitals have "sizes" that can be characterized by their expectation values of $r$ and of $r^{2}$ :

$$
\langle r\rangle_{n, l}=\frac{n^{2} a_{0}}{Z}\left[1.5-\frac{l(l+1)}{2 n^{2}}\right] ; \quad\left\langle r^{2}\right\rangle_{n, l}=\frac{n^{4} a_{0}^{2}}{Z^{2}}\left[2.5-\frac{3 l(l+1)-1}{2 n^{2}}\right]
$$

where $n$ and $l$ are the principal and angular momentum quantum numbers of the orbital and $a_{0}$ the Bohr unit of length $\left(a_{0}=0.529 \AA\right)$. These expressions can be found, for example, in ref. 7. To conceptualize the magnitude of the overlap (and thus the $\mathrm{H}_{1,2}$ coupling strength) of a Rydberg orbital with, for example, a methyl anion lone pair, an SS $\sigma^{*}$, or an amide $\pi^{*}$ orbital, think of a Rydberg s-orbital as a spherical shell of radius $\langle r\rangle_{n 0}=1.5 n^{2} a_{0} / Z$ having a radial "thickness" $\delta r$ to its electron distribution characterized by its dispersion in radial distribution $\delta r=$ $\left[\left\langle r^{2}\right\rangle_{n, 0}-\left(\langle r\rangle_{n, 0}\right)^{2}\right]^{1 / 2}=0.5 n^{2} a_{0} / Z$. This shell of thickness $\delta r$ thus has a surface area of $4 \pi 2.25 n^{4} a_{0}^{2} / Z^{2}$ and a volume of $V_{n}=4 \pi 2.25 \times 0.5 n^{6} a_{0}^{3} / Z^{3}$. In contrast, a methyl anion lone pair, an SS $\sigma^{*}$, or an amide $\pi^{*}$ orbital has a volume of $c a$. $V_{\text {bond }}=4 / 3 \pi\left(10 a_{0}\right)^{3}$. Now, consider one of the latter orbitals penetrating into a Rydberg orbital, and approximate the electron density within each of the two volumes $V_{n}$ and $V_{\text {bond }}$ as uniform. That is, within each volume, the respective wave functions are approximated by $\psi(\mathbf{r})=(1 / V)^{1 / 2}$. The $\mathrm{H}_{1,2}$ coupling should then scale with $n$ in the same manner as the overlap integral $(S)$ between the two wave functions $S=\int_{V_{\text {bond }}}\left(1 / V_{\text {bond }}^{1 / 2}\right)\left(1 / V_{n}^{1 / 2}\right) d^{3} r=\left(V_{\text {bond }}^{1 / 2} / V_{n}^{1 / 2}\right)=\sqrt{10^{3} Z^{3} / 0.5(3)(2.25) n^{6}}$ given in terms of the square root of the fraction of the volume of the Rydberg orbital that is shared with the penetrating orbital of volume $\left(10 a_{0}\right)^{3}$. Even for $n=4$, this overlap is $0.27 Z^{2 / 3}$. For $n=9, S$ is $0.02 Z^{3 / 2}$. This scaling of the overlap between a Rydberg orbital and a valence-sized orbital as $n^{-3}$ suggests that the $\mathrm{H}_{1.2}$ couplings will be small except for Rydberg orbitals in the $n=3-10$ range, not for high- $n$ Rydberg orbitals. 


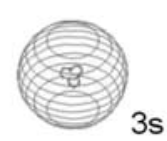

3s

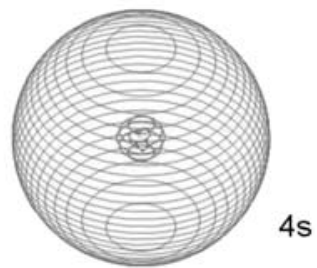

$4 \mathrm{~s}$

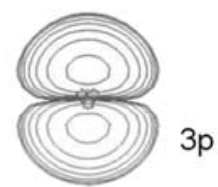

$3 p$

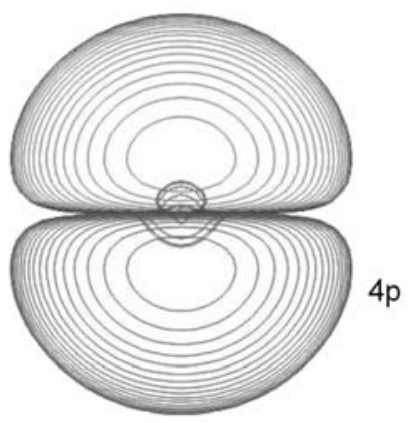

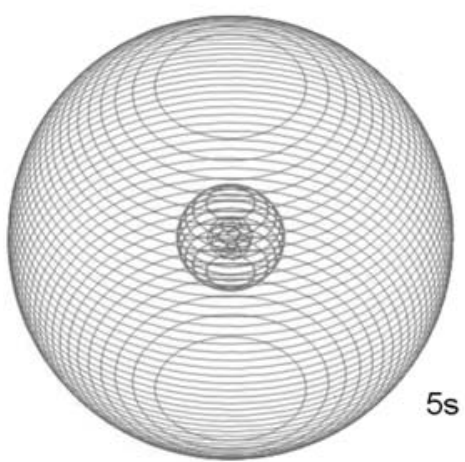

Figure 6 Plots of $3 \mathrm{~s}, 3 \mathrm{p}, 3 \mathrm{~d}, 4 \mathrm{~s}$, and $5 \mathrm{~s}$ Rydberg orbitals of $\mathrm{NH}_{4}$ with the outermost contour containing $60 \%$ of the electron density of that orbital.

have only one electron in their Rydberg orbital. However, for an anion donor such as $\mathrm{H}_{3} \mathrm{C}^{-}$, correlation is very important because the extra electron experiences very large correlations with the other methyl lone pair electron.

4. To evaluate the $\mathrm{H}_{1,2}$ couplings, one needs to carry out calculations at a very finely spaced grid (often with geometry changes along, for example, the SS bond length, of $c a .0 .01 \AA$ ) in the region of the avoided crossing. After one has determined the smallest energy gap between the two states undergoing the avoided crossing, $\mathrm{H}_{1,2}$ is taken an one-half this gap. These same calculations are what one uses to evaluate the slope difference $|\Delta F|$ entering into the LZ surface hopping probability formula.

Finally, it is important to explain the strategy that we have used to construct model compounds on which to carry out ab initio calculations from which we can gain insight into the two classes of electron transfer discussed above. For the kind of polypeptides shown in Figures 1 and 4 and for most species used in ETD or ECD experiments, the positively charged sites reside primarily on side chains that possess great motional flexibility. This means that, as the peptide undergoes 
thermal motion in the gas phase, the distances between the positive sites and any SS or OCN group will fluctuate substantially, as will the distances from one positive site to another. As a result, the Coulomb stabilization energy (Equation (2)) at the SS, OCN, and positive sites will also fluctuate with time. Ideally then, one would like to model the dynamical motions of the polypeptide's side chains and backbone and, at each instant of time, compute the rates for electron transfer from an anion donor to SS, OCN, and Rydberg sites as well as the rates of intra-peptide electron transfer. Such an ideal approach is simply not computationally feasible because of the substantial difficulties involved in each electron transfer rate calculation. Therefore, the approach we have undertaken involves:

a. Using small model compounds containing one disulfide or amide unit to limit the computational cost.

b. Fixing the distances between positive sites and SS or OCN bond sites and between positive sites in each calculation (but varying them from one calculation to another) as a way to gain data representative of that particular set of inter-site distances.

This approach allows us to generate a body of data representative of the range of geometries sampled by a polypeptide undergoing dynamical motions.

\subsection{Illustrative examples}

With the above advice and strategy in mind, we can now focus on a few illustrative cases involving electron transfer to an SS $\sigma^{*}$ orbital that subsequently affects disulfide bond cleavage as a means of further illustrating how these studies proceed and what they have told us. First, let us consider intra-peptide transfer from a Rydberg orbital on a protonated amine site, through intervening aliphatic "spacers" of varying length, to such an SS $\sigma^{*}$ orbital.

In Figure 7, we show the SS $\sigma^{*}$, excited-Rydberg, and ground-Rydberg orbitals for three model compounds ${ }^{+} \mathrm{H}_{3} \mathrm{~N}-\left(\mathrm{CH}_{2}\right)_{n}-\mathrm{S}-\mathrm{S}-\mathrm{CH}_{3}$ having $n=3,2$, or 1 from left to right.

It is important to recognize that the Rydberg orbitals have significant amplitudes in regions of space where the SS $\sigma^{*}$ orbital also does and that the degree of overlap between the Rydberg and SS $\sigma^{*}$ orbitals decreases as $n$ increases, as expected.

For $n=3$, the energy profiles of the parent compound, the species with an electron attached to the ground or excited-Rydberg orbital, and the species with an electron in the SS $\sigma^{*}$ orbital as functions of the SS bond length were shown earlier in Figure 3 where we also see the $\mathrm{H}_{1,2}$ values associated with the Rydberg SS $\sigma^{*}$ avoided crossings. Analogous data was obtained for the $n=2$ and $n=3$ cases, and the corresponding $\mathrm{H}_{1,2}$ values were obtained. When the $\ln \mathrm{H}_{1,2}$ values for ground and excited-Rydberg states are plotted for $n=1,2$, and 3 are plotted vs. the distance $R$ between the center of the SS bond and the center of charge of the Rydberg orbital, decent linear correlations are obtained as shown in Figure 8.

Such exponential decays of $\mathrm{H}_{1,2}$ with distance are characteristic of the electronic coupling strengths in all electron-transfer studies [8-11], not just those 

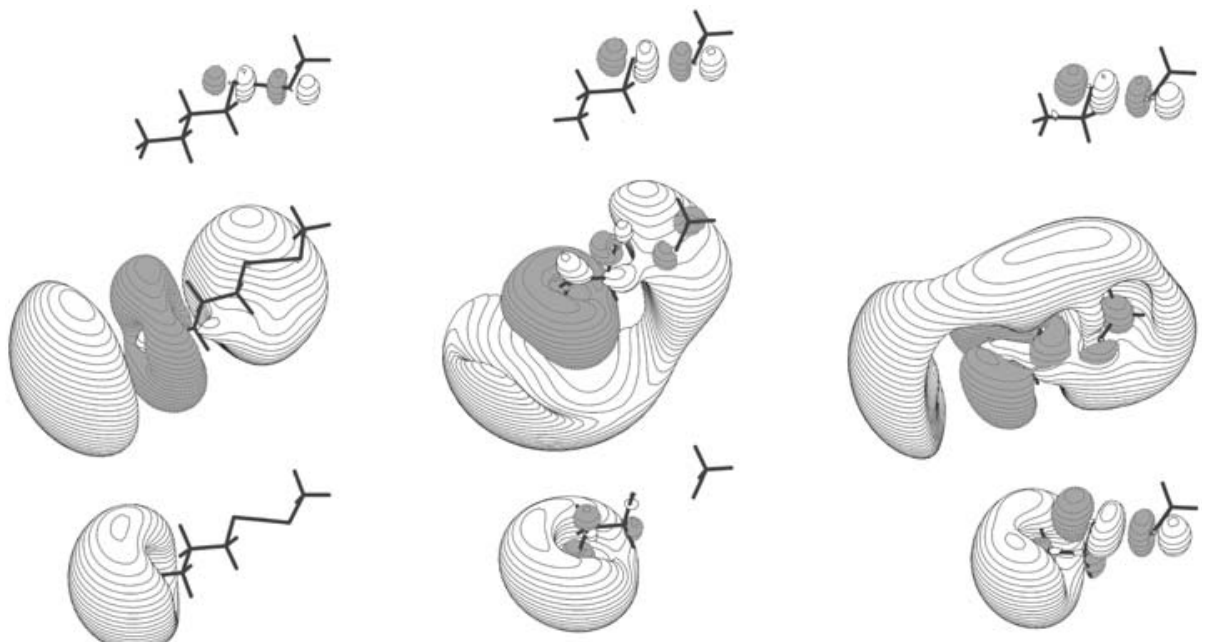

Figure 7 SS $\sigma^{*}$ (top), excited-Rydberg (middle), and ground-Rydberg (bottom) orbitals of ${ }^{+} \mathrm{H}_{3} \mathrm{~N}-\left(\mathrm{CH}_{2}\right)_{n}-\mathrm{S}-\mathrm{S}-\mathrm{CH}_{3}$ with $n=3$ (left), 2 (center), and 1 (right) (appears as Figure 5 in ref. 3s).

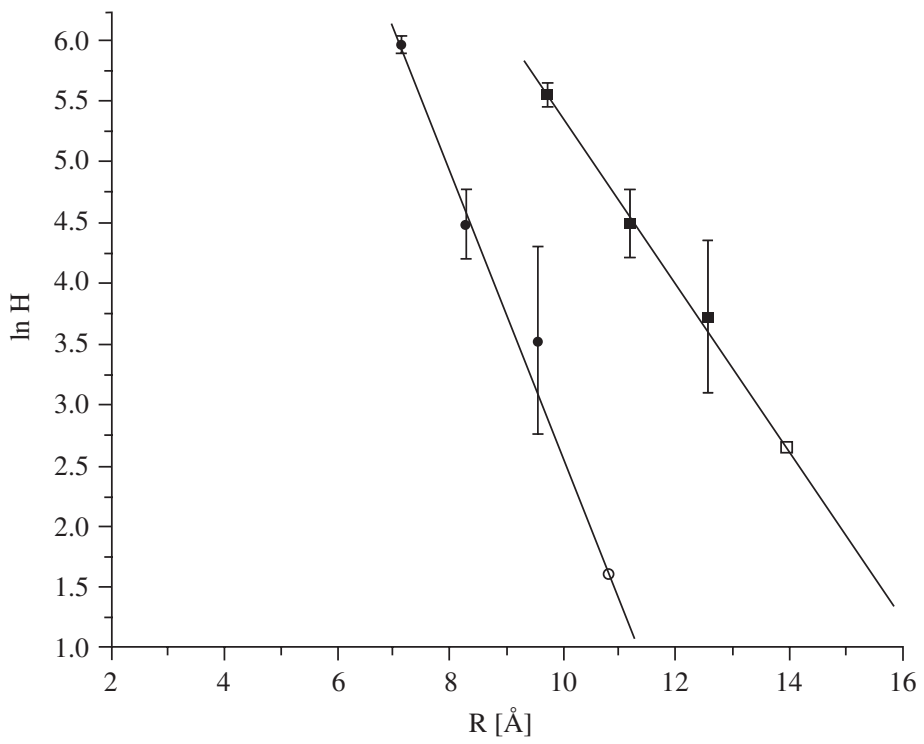

Figure 8 Plots of $\ln \mathrm{H}_{1,2}\left(\mathrm{~cm}^{-1}\right)$ vs. distance $R(\AA)$ from the center of the SS bond and the center of charge of the ground (left line) and excited (right line) Rydberg orbitals for the ${ }^{+} \mathrm{H}_{3} \mathrm{~N}-\left(\mathrm{CH}_{2}\right)_{n}-\mathrm{S}-\mathrm{S}-\mathrm{CH}_{3}$ model compounds having $n=1$, 2, and 3 (appears as Figure 6 in ref. 3s).

related to intra-peptide or anion-to-peptide electron transfer. The error bars shown in Figure 8 derive from our estimate of how small $\mathrm{H}_{1,2}$ can be before we find it too difficult to reliably determine the minimum energy splitting between two surfaces undergoing an avoided crossing. 
Although we are not able to directly determine $\mathrm{H}_{1,2}$ values as small as $0.3 \mathrm{~cm}^{-1}$ (recall, this is the smallest $\mathrm{H}_{1,2}$ that can generate an intra-peptide electron transfer that can compete with relaxations among Rydberg states), we use the near-linear plots of $\mathrm{H}_{1,2}$ vs. $R$ to extrapolate to that $R$-value where $\mathrm{H}_{1,2}^{\min }=0.3 \mathrm{~cm}^{-1}$ should be realized. For example, the data shown in Figure 8 suggest that the excited-Rydberg state can contribute to electron transfer out to $R \approx 18 \AA$, while the ground-Rydberg state can out to $R \approx 12 \AA$.

To explore whether the electron-transfer events occur primarily through-space or through-bond, we carried out calculations on model compounds in which the disulfide linkage is separated from the site of the Rydberg orbital(s) by distances similar to those arising in the studies of ${ }^{+} \mathrm{H}_{3} \mathrm{~N}-\left(\mathrm{CH}_{2}\right)_{n}-\mathrm{S}-\mathrm{S}-\mathrm{CH}_{3}$ but with no "spacer" groups between the Rydberg and SS sites. For example, we studied two model systems: $\mathrm{H}_{3} \mathrm{C}-\mathrm{SS}-\mathrm{CH}_{3}$ with an $\mathrm{NH}_{4}^{+}$ion 3-15 $\AA$ from the midpoint of the SS bond and $\mathrm{H}_{3} \mathrm{C}-\mathrm{SS}-\mathrm{CH}_{3}$ with an $\mathrm{N}\left(\mathrm{CH}_{3}\right)_{4}^{+}$ion $3-15 \AA$ from the midpoint of the SS bond. These two positive sites were chosen to model protonated amine and so-called fixed-charge sites that occur in many polypeptides. The energy profiles of the parent compound and of species with an electron attached to the SS $\sigma^{*}$, ground-, or excited-Rydberg orbitals are shown in Figures 9 and 10.

Also shown in Figures 9 and 10 are the $\mathrm{H}_{1,2}$ values (in $\mathrm{cm}^{-1}$ ) obtained by analyzing the avoided curve crossings. In Figure 11 we show plots of the natural

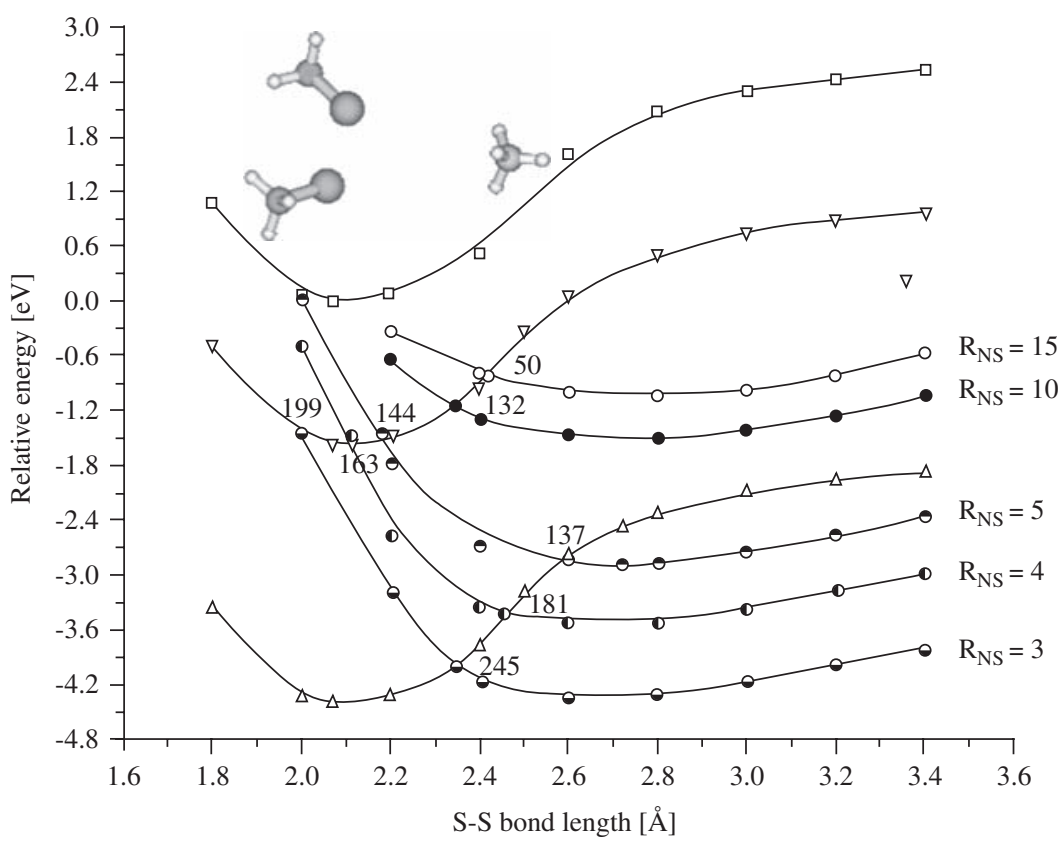

Figure 9 Energies of parent $\mathrm{H}_{3} \mathrm{C}-\mathrm{SS}-\mathrm{CH}_{3} \ldots \mathrm{NH}_{4}^{+}$(open squares), ground-Rydberg (open triangles), excited-Rydberg (inverted open triangles), and various SS $\mathrm{s}^{*}$-attached (circles) states as functions of the SS bond length, for a range of distances between the nitrogen atom and the midpoint of the SS bond (appears in Figure 8 of ref. 3s). 


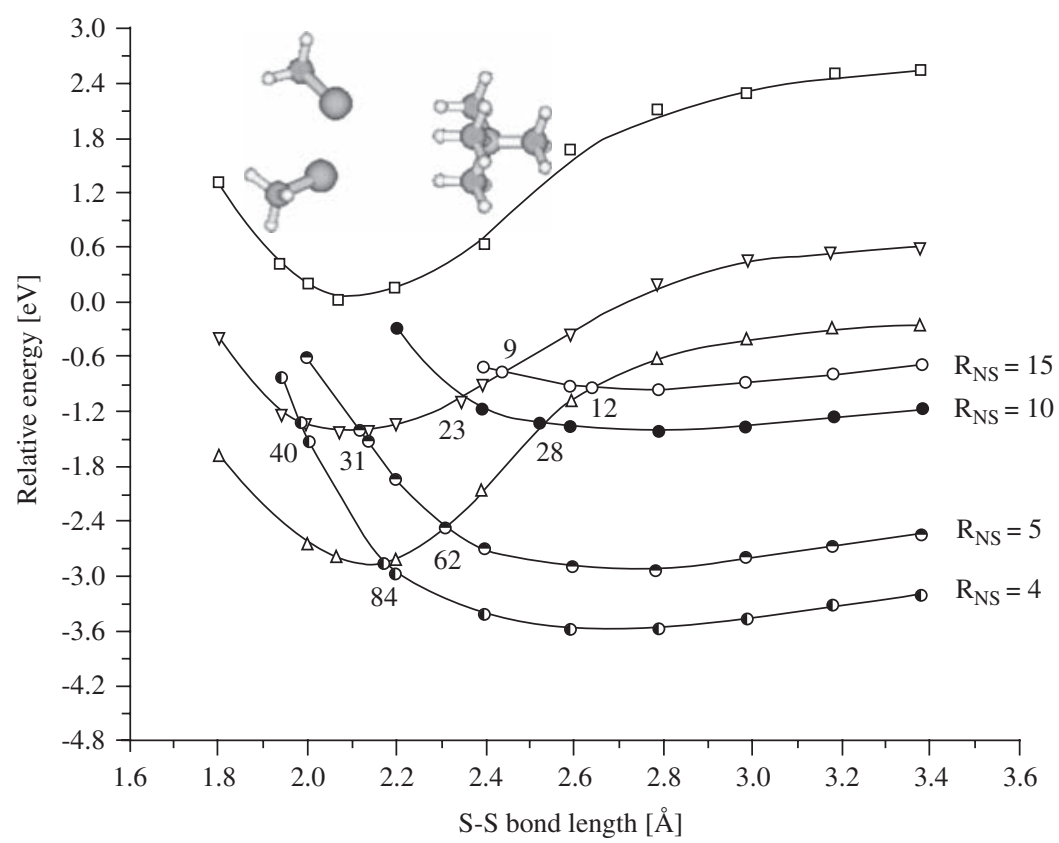

Figure 10 Energies of parent $\mathrm{H}_{3} \mathrm{C}-\mathrm{SS}-\mathrm{CH}_{3} \ldots \mathrm{N}\left(\mathrm{CH}_{3}\right)_{4}^{+}$(open squares), ground-Rydberg (open triangles), excited-Rydberg (inverted open triangles), and various SS $\mathrm{s}^{*}$-attached (circles) states as functions the SS bond length for a range of distances between the nitrogen atom and the midpoint of the SS bond (appears in Figure 8 of ref. 3s).

$\log$ of these $\mathrm{H}_{1,2}$ values as functions of the distance from the nitrogen atom to the midpoint of the SS bond for the four cases related to Figures 9 and 10.

Again, we see that the Rydberg states' couplings can extend over very large distances. Moreover, it appears (from Figures 8 and 11) that the excited-Rydberg states' coupling strength seems to decay somewhat slower with distance than those of the ground-Rydberg states. Finally, the magnitudes of the $\mathrm{H}_{1,2}$ values obtained with $-\mathrm{CH}_{2}-$ spacers present are not qualitatively larger (compare Figures 8 and 11) than those obtained in the through-space study (for a given distance). This suggests that, at least for the systems studied to date, the presence of aliphatic spacers does not qualitatively increase the rates of intra-peptide electron transfer; through-space transfer seems to be dominant.

Although space limitations preclude reviewing all of the results [3h-3u] that have come out of our studies on anion-to-peptide electron transfer and intrapeptide electron transfer, it is worth mentioning here a few of the highlights.

a. In collisions of an anion donor with a positively charged polypeptide, electron transfer to a Rydberg orbital on a positive site is 10-100 times more likely than transfer to an SS $\sigma^{*}$ or OCN $\pi^{*}$ orbital.

b. Once an electron attaches to a Rydberg orbital (probably an excited orbital), it can relax to lower-energy Rydberg orbitals in $c a .1 \mu \mathrm{s}$, or it can, in this same timeframe, undergo transfer to any an SS $\sigma^{*}$ or OCN $\pi^{*}$ orbital that is within 

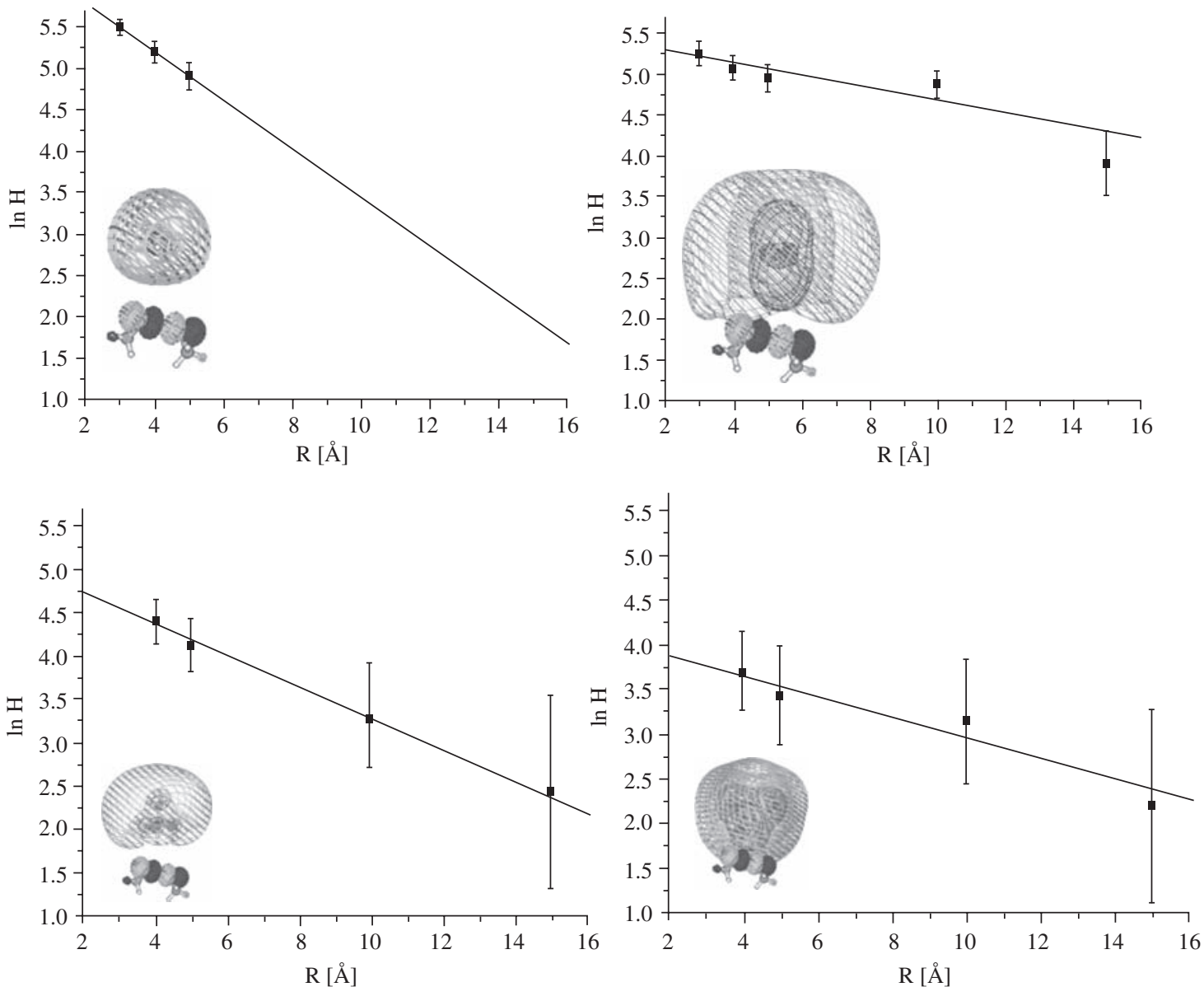

Figure 11 Plots of $\ln \mathrm{H}_{1,2}\left(\mathrm{~cm}^{-1}\right)$ vs. distance from the nitrogen atom to the midpoint of the SS bond for ground (left) and excited (right) Rydberg states of $\mathrm{NH}_{4}$ (top) and $\mathrm{N}\left(\mathrm{CH}_{3}\right)_{4}$ (bottom). Also shown are the Rydberg orbitals involved in each case along with the molecular complex's geometry (appears as Figure 7 in ref. 3p). 
$15-20 \AA$ and that is sufficiently Coulomb stabilized by nearby positive charges to render positive its electron binding energy.

c. Once an electron attaches to a Rydberg orbital, it can transfer to a Rydberg orbital on a different positive site if the two sites come within $c a .10 \AA$ of each other.

\section{RELATION TO MORE COMMON FORMS OF ELECTRON TRANSFER}

Electron-transfer processes play many very important roles in chemistry and biology. Because the present work is focused on electron-transfer events occurring within positively charged gas-phase peptides as they occur in ETD and ECD mass spectrometry experiments, it is not appropriate or feasible to review the myriad of other places electron-transfer reactions occur in chemistry. Chapter 10 of the graduate level textbook by Schatz and Ratner [12] gives a nice introduction to the main kinds of electron-transfer events that chemists usually study as well as to the theoretical underpinnings. They also give, at the end of Chapter 10, several literature references to selected seminal papers on these subjects.

In most other electron-transfer processes, one considers an electron moving from a donor (D) to an acceptor (A) through an intervening molecular structure called a bridge (B). This is much like the Rydberg-bridge-SS system treated earlier in this paper. There are then two diabatic (meaning having a fixed orbital occupancy) electronic states $\mathrm{D}-\mathrm{B}-\mathrm{A}$ and $\mathrm{D}^{+}-\mathrm{B}_{-} \mathrm{A}^{-}$of the donor-bridge-acceptor system between which one views the transfer as taking place. The energy profiles of the reactant (D-B-A) and product $\left(\mathrm{D}^{+}-\mathrm{B}-\mathrm{A}^{-}\right)$states as functions of a reaction coordinate $X$ (i.e., the direction along which the two diabatic energy hypersurfaces cross) are, in the most commonly invoked theory, represented as parabolic functions whose minima are shifted in energy by $\varepsilon_{2}-\varepsilon_{1}$ and in length along the reaction coordinate by $X_{R}-X_{L}$ as shown in Figure 12 .

The two diabatic energy profiles are expressed in terms of harmonic forms having a common force constant as:

$$
\begin{aligned}
& V_{L}(X)=\varepsilon_{1}+\frac{1}{2} k\left(X-X_{L}\right)^{2} \\
& V_{R}(X)=\varepsilon_{2}+\frac{1}{2} k\left(X-X_{R}\right)^{2}
\end{aligned}
$$

The two diabatic surfaces and wave functions are allowed to couple by way of a Hamiltonian matrix element denoted $J$ :

$$
J=\left\langle\psi_{L}|H| \psi_{R}\right\rangle
$$

and two adiabatic energy surfaces are generated from the $2 \times 2$ Hamiltonian matrix

$$
H=\left[\begin{array}{cc}
V_{L} & J \\
J & V_{R}
\end{array}\right]
$$




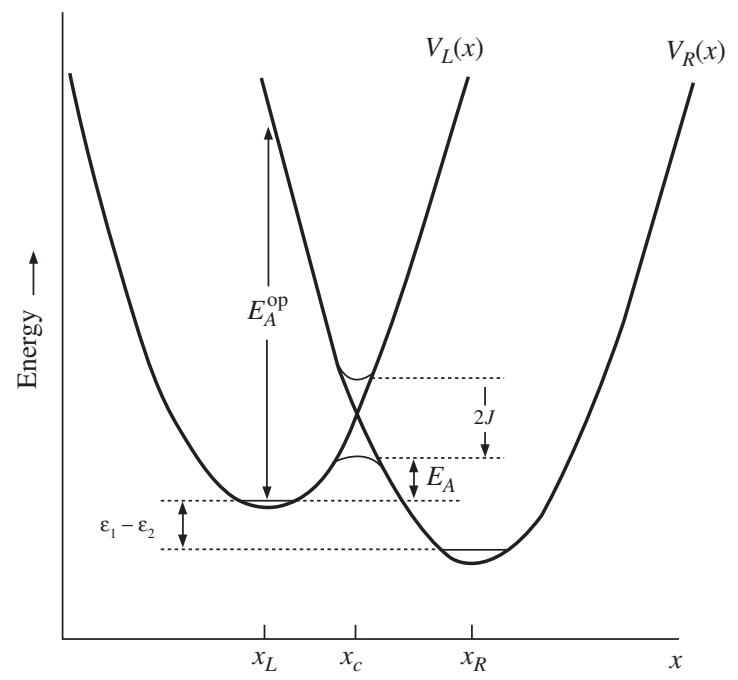

Figure 12 Plots of the energy surfaces appropriate to the D-B-A (left) and $D^{+}-B-A^{-}$(right) species as functions of the reaction coordinate along which the diabatic surfaces cross and the adiabatic surfaces undergo an avoided crossing (as shown) (appears as Figure 10.2 in ref. 12).

The two eigenvalues of this matrix

$$
E_{ \pm}=\frac{1}{2}\left[V_{L}+V_{R} \pm \sqrt{\left.\left(V_{R}-V_{L}\right)^{2}+4\right)^{2}}\right]
$$

differ by an amount $2 J$ at the point $X_{C}$ along the reaction coordinate at which the two diabatic curves cross (i.e., $V_{L}=V_{R}$ at $X_{C}$ ) as shown in Figure 12. The activation energy $E_{\mathrm{A}}$ (i.e., the energy needed to move from $\varepsilon_{1}$ to the barrier on the lower adiabatic energy surface (i.e., $\left.E_{-}\left(X_{C}\right)\right)$ ) can be expressed in terms of the so-called reorganization energy $\Lambda$ and the thermodynamic energy difference $\varepsilon_{2}-\varepsilon_{1}$ :

$$
E_{\mathrm{A}}=\frac{\left(\Lambda+\varepsilon_{2}-\varepsilon_{1}\right)^{2}}{4 \Lambda}
$$

with

$$
\Lambda=V_{R}\left(X_{L}\right)-V_{R}\left(X_{R}\right)
$$

$\Lambda$ is called the reorganization energy because (see Figure 12) it is the energy necessary to relax the system when it is in the $\mathrm{D}^{+}-\mathrm{B}_{-} \mathrm{A}^{-}$state but at the equilibrium geometry of the D-B-A state (having energy $\left.V_{R}\left(X_{L}\right)\right)$ to the energy of this $\mathrm{D}^{+}-\mathrm{B}-\mathrm{A}^{-}$state at its own equilibrium geometry.

In the cases treated in the present paper, we do not have a reorganization energy because, for example as shown in Figures 5 and 10, the two diabatic states between which electron transfer occurs (e.g., the SS $\sigma^{*}$ and excited-Rydberg states) cross so close (i.e., within the zero-point vibrational motion of the SS bond) to the minimum on the Rydberg-state surface as to render $\Lambda$ essentially zero. In more traditional electron-transfer events, $\Lambda$ contains contributions from the 
energy needed to rearrange the geometry of the D-B-A molecule itself as well as the energy needed to relax the surrounding solvent environment to the change from $\mathrm{D}-\mathrm{B}-\mathrm{A}$ to $\mathrm{D}^{+}-\mathrm{B}-\mathrm{A}^{-}$. That is, in $\mathrm{D}-\mathrm{B}-\mathrm{A}$ the surrounding solvent experiences a very different electrostatic potential than in $\mathrm{D}^{+}-\mathrm{B}-\mathrm{A}^{-}$, so the solvent molecules must reorient (and polarize) to adjust to the change in this potential. However, as noted above, in our case, there is no intramolecular reorganization energy and no solvent contribution because the mass spectroscopy experiments are carried out in the gas phase.

Returning to the more common electron-transfer cases, as shown in ref. 12, the electron-transfer rate is eventually expressed as a product of two terms. One term, which depends on the activation energy $E_{\mathrm{A}}$ in the usual $\exp \left(-E_{\mathrm{A}} / R T\right)$ manner contains the reorganization energy. The other term is proportional to $J^{2}$ and reflects the intrinsic electron-transfer rate once the system reaches the activation barrier. The scaling with $J^{2}$ arises when the couplings between the two diabatic states are treated perturbatively in this so-called nonadiabatic limit. In the cases treated in this paper, the electron-transfer rates depend on $\mathrm{H}_{1,2}^{2}\left(\mathrm{H}_{1,2}\right.$ is the same as $J)$ through the LZ expression, but we have no $\exp \left(-E_{\mathrm{A}} / R T\right)$ factor because, as already explained, our reorganization energies are essentially zero. They scale as $\mathrm{H}_{1,2}^{2}$ because, in the LZ estimate of the surface hopping probability, the two diabatic states that cross are assumed to undergo a weakly avoided crossing; that is, the LZ estimate is in line with the nonadiabatic limit discussed in conventional electron-transfer theory.

Finally, it may be useful to note that the Fermi golden rule and time correlation function expressions often used (see ref. 12, for example) to express the rates of electron transfer have been shown [13], for other classes of dynamical processes, to be equivalent to LZ estimates of these same rates. So, it should not be surprising that our approach, in which we focus on events with no reorganization energy requirement and we use LZ theory to evaluate the intrinsic rates, is closely related to the more common approach used to treat electron transfer in condensed media where the reorganization energy plays a central role in determining the rates but the $J^{2}$ factor plays a second central role.

In closing, it may be instructive to contrast the electron-transfer events taking place in polypeptides with those we have been studying relating to electrons in DNA [14]. In these studies, we simulate processes in which

a. an electron attaches to a $\pi^{*}$ orbital on one of DNA's bases, after which

b. the electron can autodetach, or

c. it can undergo a transfer through the sugar unit attached to the base and into the sugar-phosphate $\mathrm{C}-\mathrm{O} \sigma$ bond's antibonding orbital, thus leading to $\mathrm{C}-\mathrm{O}$ bond cleavage and a so-called single strand break.

The branching ratio between autodetachment and electron transfer governs the yield of strand breaks. In Figure 13, we show a qualitative depiction of the energy surfaces involved in this class of electron-transfer processes. 


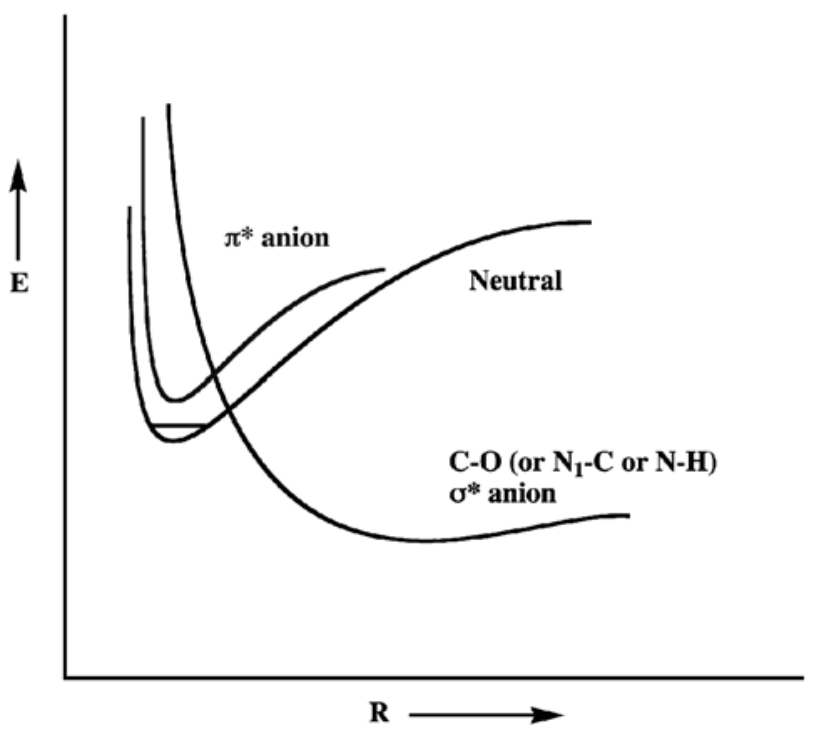

Figure 13 Qualitative depiction, as functions of the sugar-phosphate $\mathrm{C}-\mathrm{O}$ bond length, of the energy of a base-sugar-phosphate nucleotide with no electron attached (labeled neutral), with an electron attached to its base $\pi^{*}$ orbital (labeled $\pi^{*}$ anion), and with the electron residing in the sugar-phosphate $\mathrm{C}-\mathrm{O} \sigma^{*}$ orbital (lower curve) (appears as Figure 7 in ref. 14).

There are two primary differences in this DNA case when compared to the polypeptide systems discussed earlier:

1. Because the repulsive $\mathrm{C}-\mathrm{O} \sigma^{*}$-attached state crosses the base $\pi^{*}$-attached state at an energy significantly above the minimum on the $\pi^{*}$-attached state's surface (see Figure 13), the $\mathrm{C}-\mathrm{O}$ bond must undergo substantial elongation to access this crossing point. This elongation is thought to occur by thermal excitation of the $\mathrm{C}-\mathrm{O}$ stretching motion. The energy $\Delta E$ required to reach this crossing is analogous to the reorganization energy discussed earlier. This requirement gives rise to a Boltzmann $\exp (-\Delta E / R T)$ dependence in the electron-transfer rate for this DNA case, much like the reorganization energy does in the conventional electron-transfer theory discussed earlier.

2. The $\mathrm{H}_{1,2}$ matrix elements connecting the $\mathrm{C}-\mathrm{O} \sigma^{*}$-attached and the base $\pi^{*}$-attached states were found [14] to be much larger (e.g., $>1,000 \mathrm{~cm}^{-1}$ ) than in the polypeptide case (where they were usually $<300 \mathrm{~cm}^{-1}$ ). As a result, the DNA electron transfer does not occur in the nonadiabatic limit discussed earlier as it does in the polypeptides. In the DNA case, the couplings are large enough that the system evolves adiabatically (i.e., once the barrier at the crossing of the $\mathrm{C}-\mathrm{O} \sigma^{*}$-attached and the base $\pi^{*}$-attached states is reached, electron transfer is prompt) from the base to the sugar-phosphate $\mathrm{C}-\mathrm{O}$ bond that is then cleaved. 


\section{ACKNOWLEDGMENT}

This work has been supported by NSF Grant No. 0806160.

\section{REFERENCES}

1. [a] Zubarev, R.A., Kelleher, N.L., McLafferty, F.W. Electron capture dissociation of multiply charged protein cations. A nonergodic process. J. Am. Chem. Soc. 1998, 120, 3265-6. [b] Zubarev, R.A., Kruger, N.A., Fridriksson, E.K., Lewis, M.A., Horn, D.M., Carpenter, B.K., McLafferty, F.W. Electron capture dissociation of gaseous multiply-charged proteins is favored at disulfide bonds and other sites of high hydrogen atom affinity. J. Am. Chem. Soc. 1999, 121, 2857-62. [c] Zubarev, R.A., Horn, D.M., Fridriksson, E.K., Kelleher, N.L., Kruger, N.A., Lewis, M.A., Carpenter, B.K., McLafferty, F.W. Electron capture dissociation for structural characterization of multiply charged protein cations. Anal. Chem. 2000, 72, 563-73. [d] Zubarev, R.A., Haselmann, K.F., Budnik, B., Kjeldsen, F., Jensen, F. Account: towards an understanding of the mechanism of electron-capture dissociation: a historical perspective and modern ideas. Eur. J. Mass Spectrom. 2002, 8, 337-49.

2. [a] Syka, J.E.P., Coon, J.J., Schroeder, M.J., Shabanowitz, J., Hunt, D.F. A bubble-driven microfluidic transport element for bioengineering. Proc. Natl. Acad. Sci. 2004, 101, 9523-8. [b] Coon, J.J., Syka, J.E.P., Schwartz, J.C., Shabanowitz, J., Hunt, D.F. Anion dependence in the partitioning between proton and electron transfer in ion/ion reactions. Int. J. Mass Spectrom. 2004, 236, 33-42. [c] Pitteri, S.J., Chrisman, P.A., McLuckey, S.A. Electron-transfer ion/ion reactions of doubly protonated peptides: effect of elevated bath gas temperature. Anal. Chem. 2005, 77, 5662-9. [d] Gunawardena, H.P., He, M., Chrisman, P.A., Pitteri, S.J., Hogan, J.M., Hodges, B.D.M., McLuckey, S.A. Electron transfer versus proton transfer in gas-phase ion/ion reactions of polyprotonated peptides. J. Am. Chem. Soc. 2005, 127, 12627-39. [e] Gunawardena, H.P., Gorenstein, L., Erickson, D.E., Xia, Y., McLuckey, S.A. Electron transfer dissociation of multiply protonated and fixed charge disulfide linked polypeptides. Int. J. Mass Spectrom. 2007, 265, 130-8.

3. [a] Syrstad, E.A., Turecek, F. Hydrogen atom adducts to the amide bond. Generation and energetics of the amino(hydroxy)methyl radical in the gas phase. J. Phys. Chem. A 2001, 105, 11144-55. [b] Turecek, F., Syrstad, E.A. Mechanism and energetics of intramolecular hydrogen transfer in amide and peptide radicals and cation-radicals. J. Am. Chem. Soc. 2003, 125, 3353-69. [c] Turecek, F., Polasek, M., Frank, A., Sadilek, M. Transient hydrogen atom adducts to disulfides. Formation and energetics. J. Am. Chem. Soc. 2000, 122, 2361-70. [d] Syrstad, E.A., Stephens, D.D., Turecek, F. Hydrogen atom adducts to the amide bond. Generation and energetics of amide radicals in the gas phase. J. Phys. Chem. A 2003, 107, 115-26. [e] Turecek, F. NC $\alpha$ bond dissociation energies and kinetics in amide and peptide radicals. Is the dissociation a non-ergodic process? J. Am. Chem. Soc. 2003, 125, 5954-63. [f] Syrstad, E.A., Turecek, F. Toward a general mechanism of electron capture dissociation. J. Am. Soc. Mass. Spectrom. 2005, 16, 208-24. [g] Uggerud, E. Electron capture dissociation of the disulfide bond-a quantum chemical model study. Int. J. Mass Spectrom. 2004, 234, 45-50. [h] Anusiewicz, I., Berdys-Kochanska, J., Simons, J. Electron attachment step in electron capture dissociation (ECD) and electron transfer dissociation (ETD). J. Phys. Chem. A 2005, 109, 5801-13. [i] Anusiewicz, I., Berdys-Kochanska, J., Skurski, P., Simons, J. Simulating electron transfer attachment to a positively charged model peptide. J. Phys. Chem. A 2006, 110, 1261-6. [j] Sawicka, A., Skurski, P., Hudgins, R.R., Simons, J. Model calculations relevant to disulfide bond cleavage via electron capture influenced by positively charged groups. J. Phys. Chem. B 2003, 107, 13505-11. [k] Sobczyk, M., Skurski, P., Simons, J. Dissociative low-energy electron attachment to the C-S bond of $\mathrm{H}_{3} \mathrm{C}-\mathrm{SCH}_{3}$ influenced by Coulomb stabilization. Adv. Quantum Chem. 2005, 48, 239-51. [1] Sawicka, A., Berdys-Kochaska, J., Skurski, P., Simons, J. Lowenergy $(0.1 \mathrm{eV})$ electron attachment $\mathrm{S}-\mathrm{S}$ bond cleavage assisted by Coulomb stabilization. Int. J. Quantum Chem. 2005, 102, 838-46. [m] Anusiewicz, I., Berdys, J., Sobczyk, M., Sawicka, A., Skurski, P., Simons, J. Coulomb-assisted dissociative electron attachment: application to a model peptide. J. Phys. Chem. A 2005, 109, 250-8. [n] Bakken, V., Helgaker, T., Uggerud, E. Models of fragmentations induced by electron attachment to protonated peptides. Eur. J. Mass Spectrom. 
2004, 10, 625-38. [o] Skurski, P., Sobczyk, M., Jakowski, J., Simons, J. Possible mechanisms for protecting N-Ca bonds in helical peptides from electron-captue (or transfer) dissociation. Int. J. Mass Spectrom. 2007, 265, 197-212. [p] Sobczyk, M., Neff, D., Simons, J. Theoretical study of through-space and through-bond electron transfer within positively charged peptides in the gas phase. Int. J. Mass Spectrom. 2008, 269, 149-64. [q] Sobczyk, M., Simons, J. Distance dependence of through-bond electron transfer rates in electron-capture and electron-transfer dissociation. Int. J. Mass Spectrom. 2006, 253, 274-80. [r] Sobczyk, M., Simons, J. The role of excited Rydberg states in electron transfer dissociation. J. Phys. Chem. B 2006, 110, 7519-27. [s] Neff, D., Sobczyk, M., Simons, J. Through-space and through-bond electron transfer within positively charged peptides in the gas phase. Int. J. Mass Spectrom. 2008, 276, 91-101. [t] Neff, D., Simons, J. Theoretical study of electron capture dissociation of $\left[\mathrm{Mg}\left(\mathrm{H}_{2} \mathrm{O}\right)_{n}\right]^{2+}$ clusters. Int. J. Mass Spectrom. 2008, 277, 166-74. [u] Simons, J. Molecular anions. J. Phys. Chem. A 2008, 112, 6401-511. [v] Neff, D., Smuczynska, S., Simons, J. Electron shuttling in electron transfer dissociation. Inter. J. Mass Spec. 2009, 283, 122-34. [w] Neff, D., Simons, J. Analytical and computational studies of intra-molecular electron transfer pertinent to electron transfer and electron capture dissociation mass spectrometry. J. Phys. Chem. A (submitted, 2009).

4. Gutowski, M., Simons, J. Lifetimes of electronically metastable double-Rydberg anions: $\mathrm{FH}_{2}^{-}$. J. Chem. Phys. 1990, 93, 3874-80.

5. Simons, J., Gutowski, M. Double-Rydberg molecular anions. Chem. Rev. 1991, 91, 669-77.

6. Skurski, P., Gutowski, M., Simons, J. How to choose a one-electron basis set to reliably describe a dipole-bound anion. Int. J. Quantum Chem. 2000, 80, 1024-38.

7. Pauling, L., Wilson, E.B. Jr. Introduction to Quantum Mechanics with Applications to Chemistry, Dover Publications, New York, 1985.

8. McConnell, H.M. Intramolecular charge transfer in aromatic free radicals. J. Chem. Phys. 1961, $35,508$.

9. Mujica, V., Kemp, M., Ratner, M.A. Electron conduction in molecular wires. II. Application to scanning tunneling microscopy. J. Chem. Phys. 1994, 101, 6856.

10. Jordan, K.D., Paddon-Row, M.N. Long-range interactions in a series of rigid nonconjugated dienes. 1. Distance dependence of the $\pi_{+}, \pi_{-}$and $\pi_{+}^{*}, \pi_{-}^{*}$ splittings determined by ab initio calculations. J. Phys. Chem. 1992, 96, 1188.

11. Curtiss, L.A., Naleway, C.A., Miller, J.R. Superexchange pathway calculation of long-distance electronic coupling in $\mathrm{H}_{2} \mathrm{C}\left(\mathrm{CH}_{2}\right)_{\mathrm{m}-2} \mathrm{CH}_{2}$ chains. Chem. Phys. 1993, 176, 387.

12. Schatz, G.C., Ratner, M.A. Quantum Mechanics in Chemistry, Prentice Hall, Englewood Cliffs, NJ, 1993.

13. Taylor, H., Simons, J. A different view of molecular electronic transitions. J. Phys. Chem. 1986, 90, 580-3.

14. Simons, J. How do low-energy $(0.1-2 \mathrm{eV})$ electrons cause DNA strand breaks? Acc. Chem. Res. 2006, 39, 772-9. 
accelerated molecular dynamics, 79

acceptor, 178

activation energy $\mathrm{E}_{\mathrm{A}}, 180$

ADME-Tox, 102-105, 109-110, 112, 114, 115, $117-120,122,123$

aliphatic "spacers", 173

anion-to-peptide electron transfer, 174

Atomic orbital basis sets, 170

basin constrained molecular dynamics, 85

bias potential, 83,91

bias strength, 91

block averaging, 31

blood-brain barrier, 102, 109, 122

bond-boost potential, 91

bridge, 178

Caco-2 absorption, 108

CC-R12 with connected singles and doubles (CCSD-R12), 133, 138, 141

CC-R12 with connected singles, doubles, and triples (CCSDT-R12), 133, 139, 141

$\operatorname{CCSD}(2)_{\mathrm{R} 12}, 140$

CCSDTQ-R12, 133, 141

cholesterol, 5, 8, 10, 15

collision-induced dissociation, 164

complementary auxiliary basis set (CABS), 137

complete active space self-consistent field, 150

computer algebra, 141

concerted rotations, 51, 64

convergence, 23

correlation function, 25, 33, 138

correlation time, 32

correlations, 30

Coulomb attraction, 165

Coulomb potential, 168

Coulomb stabilization energy, 173

coupled-cluster (CC) theory, 132

couplings, 166

$\mathrm{Cu}(100), 95$

cusp condition, 134

DAPC, 8,9

DDPC, 11

density matrix renormalization group, 150, 151 diabatic energy hypersurfaces, 178

diffuse functions, 170

disulfide, 164

DLPC, 8, 11, 12, 15

DMPC, 11, 12, 15

DMSO, 14

donor, 178

DOPC, 4, 8, 12, 13, 15

DPPC, 8, 10-12, 13-15

DPPS, 11

DSPC, 11

effective sample size, 37

electron correlation, 150

electron transfer, 167

electron-capture dissociation (ECD), 164, 165

electronic coupling, 169

electrons in DNA, 181

electron-transfer dissociation (ETD), 164

electron-transfer, 166

ensemble averages, 31

EOM-CCR12, 143

equations, 143

equilibrium ensemble, 24

ergodicity, 29

error analysis; principal component; block averaging, 24

error estimation, 23, 31

excited states, 171

explicitly correlated methods, 133

explicitly correlated second-order

Møller-Plesset, 133

exponential decays, 174

F12 methods, 133

Fermi golden rule, 180

first-order cusp condition, 140

first-order kinetics, 81

FLIP-FLOP, 6, 12, 14-16

free energy, $9,11,15$

geminal excitation operator, 136

generalized ensemble, 69

harmonic transition state theory, 85

$2 \mathrm{H}_{1,2}, 166$

Hartree-Fock, 171 
HMPC, 11

human intestinal absorption, 102-104, 111, 124

Hylleraas functional, 136

hyperdynamics, 83

ijkl ansatz, 137

implicit solvation, 51, 55, 67

importance sampling, 51

index-permutation symmetry, 141

infrared multiphoton dissociation, 164

infrequent events, 79

intra-peptide, 174

intra-peptide electron transfer, 167

Landau-Zener (LZ) theory, 166

lipid bilayers, 4

many-body basis, 150, 155-156, 161

MARTINI model, 7, 9

matrix product states, 150

MD simulations, 7-8, 12

membrane, 4,6

molecular dynamics, 79

Møller-Plesset perturbation theory, 171

Monte Carlo, 50-54, 56, 58, 60, 62, 64, 66-68, 70-71

MP2 method, 133

MP2-R12, 135

$\mathrm{N}-\mathrm{C}_{\alpha}, 164$

non-dynamic correlation, 149-150, 152-153

oral bioavailability, 102-105, 114-116, 119-120, 123

orthogonality projector, 136

parallel-replica dynamics, 81

phospholipid, 8

plasma protein binding, 102-104, 116-117, 122,124

POPC, 9, 12, 15

pores, $6,12,14$

post Hartree-Fock methods, 149

potential of mean force, 92
QSAR, 103, 105-106, 108, 110-111, 116-119, $121,123-124$

R12 method, 133, 134

radiationless relaxation, 167

reorganization energy, 179-180

replica exchange, 31

resolution of the identity (RI), 133

RI approximation, 137

Rydberg states, 165

sampling Quality, 23

SDPC, 8

second-order Møller-Plesset perturbation (MP2-R12), 133

self-learning hyperdynamics, 89

Slater-type correlation function, 133, 138

solid-liquid interface, 89

solubility, 102-108, 112, 114-116, 120, 122-123

spatial parallelization, 93

spatially parallel temperature accelerated dynamics, 93

special basis functions, 171

standard approximation, 137

standard error, 34

statistical uncertainty, 25

strongly correlated electrons, 150

structural histogram, 39

superstate parallel-replica dynamics, 88

synchronous sublattice algorithm, 94

temperature-accelerated dynamics, 85

temporal parallelization, 81

thin film growth, 95

through-space or through-bond, 175

time correlation function, 180

timescale separation, 88

timescales, 25

transition state theory, 83

umbrella sampling, 9, 11

variance, 25 
${ }^{12} \mathrm{C}^{16} \mathrm{O}_{2}, 3,168$

3D QSAR, 2 , 182; $\underline{3}, 67,71$

$\pi-\pi$ interactions, $\underline{3}, 183$

ab initio, 3, 215, 219, 220

ab initio modelling, 1, 187, 188

ab initio thermochemical methods, 1 , 33, 37, 45

absorption, 5, 103, 108-113, 121-123

intestinal, 1, 137-138

see also ADMET properties

accelerated molecular dynamics, 2, 230

ACPF, $\underline{3}, 163$

action optimization, 3 , 17, 19

activated state, $\underline{3}, 220-222$

active database, $\underline{3}, 157$

Active Thermochemical Tables, $\underline{3}, 159$

active transport, 1 , 139, 140

acyl carrier protein synthase (AcpS), 1, 179

adenosine triphosphate (ATP) site recognition, 1 , 187, 188

adiabatic approximations, 1, 20, 25, 27

adiabatic Jacobi correction (AJC), 3, 158

ADME-Tox, ㅁ, 101-104, 108-109, 11̄1, 113, 114, $116-119,121,122$

ADMET properties

active transport, $1,139,140$

aqueous solubility, $\underline{1}$, 135-137, 162

blood-brain barrier permeation, $\underline{1}, 140-142$

computational prediction, 1 , 133-151

cytochrome P450 interactions, 1 , 143, 144

drug discovery, 1, 159-162

efflux by P-glycoprotein, 1, 140, 160, 161

intestinal absorption, $1,1 \overline{3} 7,138$

intestinal permeability, $1,134,135,161$

metabolic stability, $1,142,143,162$

oral bioavailability, $\underline{1}, 134,138,139,159$, 160

plasma protein binding, 1,142

toxicity, 1,144

AGC group of kinases, 1,196

agrochemicals, 1,163

AK peptide, $2, \overline{9} 1$

"alchemical" free energy transformations, 3, 41-53

alignment-independent molecular descriptors, $\underline{3}, 69$
AMBER, 2, 91

AMBER force fields, $1,92,94-97,99,119-121$

angular wavefunctions, $1,225-228$

anisotropic polarizability tensors, $\underline{3}, 180$

ANO basis, $\underline{3}, 201$

apparent errors, $\underline{3}, 196$

applicability domain, 2, 113, 118, 120, 123, 125

aqueous solubility, 1, 135-137, 162

aromatic cluster, $\underline{3}, \overline{2} 12,221$

assay, 4, 23, 24, 204, 205, 208, 210, 212, 213, $221,223,225,226,229,230,232-235,238$, 239

asymmetric top notation, 3 , 159

atomic orbital representations, 1 , 225-228

atomistic simulation

boundary conditions, 1,80

experimental agreement, $1,77,78$

force fields, 1 , 77, 79-82

methodological advances, 1,79

nucleic acids, 1, 75-89

predictive insights, $1,78,79$

sampling limitations, $\underline{1}, 80-82$

atomistic simulations

time scale, 3, 15

transition path methods, 3, 16

ATP see adenosine triphosphate

aug-cc-pVnZ, 3 , 198

AUTODOCK, $\underline{1}, 122,123 ; \underline{2}, 184$

B-factors, $\underline{3}$, 32, 34, 35

B3LYP functional, 1 , 32, 48-50

back-propagation neural networks (BPNN),

Bad, $\underline{2}, 136,137$

bagging, 2, 136

Bak, 2, 197, 198, 203-205

barrier heights, 2, 64, 73

base pair opening, 1,77

basis set superposition errors (BSSE), 2, 68, $74,76,78$

basis sets, 1 , 13-15, 32, 33; $\underline{3}, 195$

Bax, 2, 197, 198, 203, 204

Bayes model, 2, 157

Bayesian methods, 2, 132

Bcl-2, 2, 197, 198, 201, 203-206

Bcl-xL, 2, 197, 203-206

Bennett acceptance ratio, $\underline{3}, 44,45$ 
benzene dimers, 3, 188

benzene-water, 3 , 186

Bessel-DVR, 3 , 167

Betanova, $1, \overline{2} 48-9$

Bethe-Salpeter equation, 1, 27

bias potential, 2, 224-226, 229, 230

Bid, 2, 197, 203, 205

Bim, 2, 197, 203

binding affinities, $\underline{1}, 78$

binding free energy, $4,69,73,81,82,164$ calculating, 1, 114-119

protein-ligand interactions, $1,113-130$

scoring functions, 1, 119-126

binding rate, $\underline{4}, 74-8 \overline{2}$

bioavailability, 1 , 134, 138, 139, 159, 160; $\underline{5}$, 103, 104, 113-119, 121, 122

bioinformatics, 4, 4, 12, 18, 30, 33, 68, 206

biological activity, 4, 24, 204-206, 209, 210, $212,213,218, \overline{2} 19,227,232$

bio-molecular simulation atomistic simulation, 1, 75-82 nonequilibrium approaches, 1,108 protein force fields, $1,91-102$ protein-ligand interactions, $1,113-130$ water models, 1, 59-74

biospectrum similarity, 2,150

Bleep, 2, 162

block averaging, 푸 31, 33-37, 44, 47, 61

blood-brain-barrier, $\underline{5}, 109,110,122$

blood-brain barrier permeation, $1,140-142$, 160, 161

BO approximation, $\underline{3}, 158$

body-fixed frame, $\underline{3}, 166$

bond breaking configuration interaction, 1,51 coupled cluster methods, $\underline{\overline{1}}, 52,53$ generalized valence bond method, $1,47,48$ Hartree-Fock theory, 1, 46, 48-51 multireference methods, $1,51-53$ perturbation theory, $1,51,52$ potential energy surface, 1,54 quantum mechanics, $1,45-56$ self-consistent field methods, 1 , 46, 47, 53 spin-flip methods, 1,53

bond vector(s), $3,167,168$

boost energy, 2, 225-227

boosting, 2, 136, 151

Born-Oppenheimer approximation, 1, 3, 54

Born-Oppenheimer (BO), $\underline{3}, 156$

BOSS program, 2, 264

boundary conditions, 1,80

Boyer Commission, 1, 206-207

BPNN see back-propagation neural networks

Bragg's Law, $\underline{3}$, 89, 90, 97

Breit, $\underline{3}, 164$
Breit term, 3, 163

Bridgman täbles, 1, 224

BSSE see basis set superposition errors

Brownian dynamics, $\underline{4}, 77$

Caco-2 absorption, 프, 102

CAMK group of kinases, $1,186,196$

Carnegie Foundation, 1, 206-207

casein kinase 2 (CK2), $\overline{1}, 197$

Casida's equations, $\underline{1}, \overline{21}, 22,25$

caspase-3, 2, 206

caspase-9, ㄱ, 206, 208

CASSCF see complete-active-space selfconsistent field

CATS3D, 2, 149

catalysis, $\underline{4}, 97,155-157,161$

CBS- $n$ methods, 1 , 36, 37

CC see coupled cluster cc-pCVnZ, 3, 198, 199

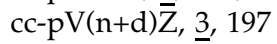

cc-pVnZ, 3, 196, 199, 202

cc-pVnZ-DK, $\underline{3}, 200,202$

cc-pVnz-PP, 3 , 201, 202

cc-pwCVnZ, 3 , 198, 199

$\operatorname{CCSD}(\mathrm{T}), \underline{3}, \overline{160}$

CD see circular dichroism

CDKs see cyclin-dependent kinases

central nervous system (CNS) drugs, 1, 160, 161

$\mathrm{CH}_{2}$ radical, $\underline{3}, 156$

chance correlations, 2,153

charge transfer (CT), 1,26

charge transfer interactions, 3,180

CHARMM force fields, $1,77,79,92-95$, 97-99, 119, 120

chemical amplification, 2, 11

chemical Kinetics Simulator, 2, 4

Chemical Markup Language (CML), 3, 116, 126

chemical space (size of), $\underline{2}, 143$

chemical structures, $4,12 \overline{8}, 204,205,208,211$, 218-220, 224, 230 , 234

chemical vapor deposition (CVD), 1, 232, 233

chemScore, 2, 162

cholesterol, $\overline{5}, 5,6,8-12,15,16$

circular dichroism (CD) spectra, 1, 22-24

circular fingerprints, 2 , 144

cis-trans isomerization, 2, 228, 229

CI see configurational interaction

classification, 4 14 , 15, 17, 27, 44-57, 212, 239

cluster-based computing, 1,113

CMAP see correction maps

CMGC group of kinases, 1 , 186, 192-194

CNS see central nervous system 
$\mathrm{CO}_{2}, \underline{3}, 162,168$

coarse-graining, $\underline{4}, 111$

cold shock proteins (CSP), 3, 24

combinatorial QSAR, 2, 113, 120

CoMFA, 2, 152

compartmentalization, 2, 11

complete basis set, 3,196

complete basis set (CBS) full configuration interaction (FCI), 3 , 156

complete-active-space self-consistent field (CASSCF) method, 1, 47, 53

compound equity, 1,171

computational protein design (CPD), 1 , 245-253

degrees of freedom, 1,246

energy function, $1,2 \overline{4} 6,247$

examples, 1, 248--250

search methods, 1 , 247, 248

solvation and patterning, 1, 247

target structures, 1 , 246

computational thermochemistry

ab initio methods, $1,33,37,45$

CBS- $n$ methods, $1,36,37$

density functional theory, 1 , 32, 33

empirical corrections, $1,34-36$

explicitly correlated méthods, 1,39

G1, G2, G3 theory, 1, 34-36

hybrid extrapolation/correction, 1, 36-37

isodesmic/isogyric reactions, $1,3 \overrightarrow{4}$

nonempirical extrapolation, $1,37-39$

quantum mechanics, $1,31-4 \overline{3}$

semi-empirical methods, 1 , 31, 32

Weizmann- $n$ theory, $1,37-39$

concerted rotations, $\underline{5}, \overline{63}, 65$

configurational interaction (CI), 1, 9, 10, 48, 51

configurational space, 2, 84

conformation change(s), 3, 32-36

conformational changes, substrate induced P450, 2, 173

conformational flexibility, 1,173

conformational flooding, 2, 221, 223, 224

conformational fluctuations, $\underline{4}, 74,81,109$, 161

conformation restraints, $\underline{3}, 49,50$

conformational sampling, $\underline{3}, 48,49$

conformational Transitions, 2, 221, 222, 227

consensus approaches, $1,14 \overline{5}$

consensus scoring, 2, 158

continuum salvation models, 3 , 198, 203

convergence, $\underline{5}, 26,27,37-41, \overline{68}, 92,132,143$, 144,156

core correlation, $\underline{3}, 198,203$

core-valence, $3,1999,202$

correction maps (CMAP), 1, 95, 96, 98 correlating functions, 3, 197

correlation energy, $2, \overline{53}, 54,59-62,64-71,73$, 74,76

correlation methods, $\underline{1}, 8-11$

correlation-consistent, 3, 160, 196

Council for Chemical Research, 1, 240

Council on Undergraduate Research (CUR), 1, 206-208

coupled cluster (CC) methods, $\underline{1}, 10-11$, $37-40,48-50,52,53 ; 5,131,132$

CPD see computational protein design

CPHMD, 3, 6

Crooks relationship, $\underline{3}, 45$

cross-validation

leave-group-out, 3,67

leave-one-out, $3, \overline{6} 7$

Crystallographic Courseware, $\underline{3}, 96$

CT see charge transfer

$\mathrm{Cu}, \mathrm{Zn}$ superoxide dismutase (SOD), 3, 24, 25

CUR see Council on Undergraduate Research current density, 1, 27

curvilinear, $\underline{3}, 27$

CVD see chemical vapor deposition

cyclin-dependent kinases (CDKs), 1, 186, 192-194

CVRQD, 3, 161-164

CYP inhibitor, 3 , 65,71

CYP substrate, $\overline{3}, 65,71$

cytochrome c, 3, 22

cytochrome $\mathrm{P} 4 \overline{5} 0,2,171 ; \underline{3}, 63,64$

2C5, 2, 172

$2 \mathrm{C} 9, \overline{2}, 172$

$3 \mathrm{~A} 4, \overline{2}, 172$

BM-3, 2, 174

eryF, 2, 174

terp, $\underline{2}, 174$

cytochrome P450 interactions, $\underline{1}$, 143, 144

D-Score, 2, 161

D/ERY motif, $\underline{3}, 211$

D2.50, 3, 211

D\&C see divide and conquer

DA see discriminant analysis

data analysis, $\underline{4}$, 42, 218, 223, 226, 227, 232, 239

database, 3 , 169; $\underline{4}, 10,13,17,24-26,49-52,68$, 92, 204-213, 218, 220-226, 228, 236, 238, 239

database mining, 2, 114, 121-125

databases

drug-likeness, $\underline{1}$, 155, 156

ligand-based screening, 1, 172-175

self-extracting, $1,223,22 \overline{5}$

symbolic computation engines, 1 , 223-225 
data-mining, 4, 205, 206

Davidson correction, 3,163

DBOC, 3, 160, 163

de novo protein design, 1, 245

dead-end elimination (DEE), 1, 247-249

degrees of freedom, 1, 246

density fitting, 2, 55, 74,77

density functional theory (DFT)

bond breaking, 1, 48, 49

computational thermochemistry, 1 , 32, 33

protein-ligand interactions, $\underline{1}, 11 \overline{6}$

state of the art, 1, 4, 11-15

time-dependent, 1, 20-30

descriptor binarization effect, 2, 152

designability, $\underline{4}, 7,9,11,13,17$

DEWE, 3, 168

DEZYMER algorithm, 1,249

DF-LCCSD(T), 2, 55

DF-LMP2, 2, 55, 73, 75

DFT see density functional theory discriminant analysis (DA), 1, 138

diagonal Born-Oppenheimer corrections (DBOC), 3, 158

dielectric constant, $4,73,74,97,98,100$, 109-111, 113-115, 117, 128, 129, 133

diffusion, $4,75,77,79,82,140,141,147-152$, 174, 176-180, 183, 184, 196

digital repository, 3, 103, 107, 108, 125, 129

dipole polarizability, $\underline{3}, 179$

discrete path sampling (DPS), $\underline{3}, 16$

discrete variable representation (DVR), $\underline{3}, 166$

displacement coordinates, $\underline{3}, 168$

dissipative MD, 3 , 139

distant pairs, 2 , $54,62,63$

distributed computing, 1,113

distributed multipolar expansion, 3, 179

distribution see ADMET properties

divide and conquer (D\&C) algorithm, $\underline{1}$, 116-117

DKH, 3, 200

DMS, $\overline{3}, 156$

DMSs, $\underline{3}, 163,165$

DNA gyrase, 2, 280

DOCK, 2, 157, 159, 161, 179, 184-186, 299-303, 308, 314-317, 319-320

DOCK program, 1, 173, 174, 177, 178, 189

docking, 1 , 79, 114, 119, 121, 155, 169, 172-174, $178,189-196 ; 2,141,145,157,159,161$, $162,284,297-303,305-307,309,311$, $313-321,323 ; 4,27,68,82,160,161,207$, 212

DockIt, 2, 299, 300, 317

DockScore, 2, 161

DockVision, 2, 299, 300, 315-317

domain approximation, 2, 53, 64, 73-76, 78 domain extensions, 2, 54, 59, 62, 63, 77

DOPI, 3, 166, 168

drug discovery, $1,155-168 ; \underline{3}, 64$

agrochemicals, 1,163

aqueous solubility, 1,162

chemistry quality, 1,157

CMS drugs, $1,160,161$

databases, $\underline{1}, 155,156$

drug-likeness, 1, 155-157

intestinal permeability, $\underline{1}, 161$

lead-likeness, 1, 159

metabolic stability, 1,162

oral drug activity, 1, 159-160

positive desirable chemistry filters, $\underline{1}, 158$, 159

promiscuous compounds, $\underline{1}, 162,163$

druggability, 4, 23, 29-33, $213^{-1}$

drug-drug interactions, 3,63

drug-likeness, 1, 155-157; 2, 160

DrugScore, 2, 161,162

Dublin-core metadata (DC), $\underline{3}, 104,107,108$, 125

DVR, $\underline{3}, 167$

E6.30, 조 211

Eckart-Watson Hamiltonians, $\underline{3}, 167$

education

research-based experiences, $1,205-214$

stochastic models, $1,215-220$

symbolic computation engines, $1,221-235$

effective core potentials, $\underline{3}, 200$

effective fragment potential (EFP), $\underline{3}, 178$

efflux by P-glycoprotein, $1,140,160,161$

EFP, 2, 267; 3, 178, 190

EFP-QM, $\overline{3}, 182$

$\mathrm{EFP} / \mathrm{PCM}, \underline{3}, 181$

induced dipolses, 3,181

elastic network modēl(s), 3, 31-37

electron capture dissociation, 5,164

electron correlation methods, $\overline{1}, 8-11$

electron transfer, $\underline{5}, 164,165-1 \overline{7} 0,172-176$, 178-181

electron transfer dissociation, 5, 164

electronic embedding, 2, 37

electronic Schrödinger equation, 1, 3-15

electrostatic interaction, 3,179

empirical force fields, $\underline{1}, \overline{9} 1-102$

empirical PESs, $\underline{3}, 164$

empirical scoring functions, $1,122,123$

energy function, $1,246-247$

enrichment, 2 , 297, 302, 303, 305-309, 313-319

enzyme, 4, 6, 25, 27, 32, 96, 97, 155-165, 208

error analysis, $\underline{5}, 24$

Essential dynamics, 2, 233, 236, 242-244, 247 
Euler angles, 3, 168

evolutionary determinants, $\underline{4}, 4,5$

evolvability, $\underline{4}, 7-9,17$

Ewald summation, 2, 265

Ewald summation techniques, 1, 59, 62, 75

exact exchange, $1,26,27$

exchange repulsion, $3,179,180$

excited state structure/dynamics, 1, 24

excretion see ADMET properties

explicit- $r 12$ correlation, $5,132,140$

explicit solvent, $2,98,9 \overline{9}, 101,102,104-106$

exponential damping functions, $\underline{3}, 180$

extended systems, 1 , 26

extensible metadata platform (XMP), 3, 104, 107, 109-111

\section{F-Score, 2, 161}

FCI, $\underline{3}, 1 \overline{6} 0$

feature selection, 2, 151, 153

FEP see free energy perturbation

FEPOPS, 2, 146

few-body systems, $\underline{3}, 158$

few-electron systems, 3,156

Fingal, 2, 148

fitness density, 4, 11, 14, 17

first-principles thermochemistry, $\underline{3}, 160$

FIS3, 3, 161, 162, 164

FKBP, 3, 52

FlexX, 1 , 173, 178, 189; 2, 157, 159, 184, 186, $29 \overline{9}, 300,308,313-\overline{3} 19$

$\mathrm{Flo}+299,300,317$

FLO99, 1, 178

Florida Memorial College, 1, 212

fluctuation theorem, 1, 109

fluid properties, 1 , $239-244$

focal-point approach (FPA), 1, 39; 3,160

folding intermediate states, $\underline{3}, 9$

force fields, 3, 162

molecular simulations, 1, 239, 240

nucleic acids, 1 , 77, 79-8 2

protein-ligand interactions, $\underline{1}, 116,119-121$

proteins, 1, 91-102

structure-based lead optimization, $\underline{1}, 177$

FPA, $\underline{3}, 160$

fragment positioning, $1,175-177$

FRED, 2, 148, 161, 299, 300, 313, 314, 317, 319

free energy, 1, 96, 103-111, 113-130; 4, 6, 69,

73, 92, 108-111, 115, 117, 127-129, 132,

$133,157,163,164,181,182,187 ; \underline{5}, 6-16$, 55,109

free energy calculations, 3, 41-53

free energy perturbation (FEP), $1,104,106 ; \underline{2}$, 265 functional microdomains, 3, 211

Fuzzy clustering, 2, 160

fuzzy logic, $\underline{1}, 218$

G-protein coupled receptors (GPCRs), $\underline{3}, 209$

G-Score, 1, 123; 2, 161

G1, G2, G3 theory, 1 , 34-36

GAMESS, 3, 190

Gaussian Geminal Methods, 2, 25

Gaussian quadratures, $\underline{3}, 166$

GB-1 beta hairpin, 2, 91, 92

generalized Born, 2 , 222; $\underline{4}, 73,109,110,115$, $117,126,129,131,13 \overline{4}$

generalized conductor-like screening model (GCOSMO), 2, 266

generalized finite $\bar{b}$ asis representation (GFBR), $\underline{3}, 167$

generalized gradient approximation (GGA), 1,12

generalized valence bond (GVB) method, 1 , $47-48$

Ghose/Crippen descriptors, 2, 160

Glide, 2, 161, 299, 300, 302, 303, 313-319

global matrices, 1 , 116-117

glutathione peroxidase, 2,47

GOLD, 2, 161, 162, 184-186, 299, 300, 313-319

GRAFS, $\underline{3}, 210$

graphical representations, 1, 225-228, 232, 233

GRID, 2, 148-149

GRIND, 2, 148

GROMAC̄S, 2, 89, 91

GROMOS, 2, 91

GROMOS force fields, 1,97

GVB see generalized valence bond

$[\mathrm{H}, \mathrm{C}, \mathrm{N}], \underline{3}, 163$

$\mathrm{H}_{2}, 3,158$

$\mathrm{H}_{2}^{+}$-like systems, $\underline{3}, 158$

$\mathrm{H}_{2}^{16} \mathrm{O}, 3,160,164$

$\mathrm{H}_{2}^{17} \mathrm{O}, \overline{3}, 159,160,164$

$\mathrm{H}_{2}^{18} \mathrm{O}, \overline{3}, 164$

$\mathrm{H}_{2} \mathrm{O}, \underline{3}, 162,163,168$

$\mathrm{H}_{2} \mathrm{~S}, \underline{3}, 163$

$\mathrm{H}_{2}^{+}, 3,158$

Hartree-Fock (HF), $\underline{3}, 160$

Hartree-Fock (HF) method, 1, 4-11, 13-15, 20, $21,46,48-51$

HDM2, 2, 209

HEAT (High-accuracy Extrapolate $A b$ initio Thermochemistry), 3, 160

Hellmann-Feynman theorem, 1, 21

HF limit, 3, 197 
hierarchical protein design, 1, 245

high throughput docking (HTD), 2, 298-302, 304-306, 308, 309, 317-320

high-resolution spectra, $\underline{3}, 157$

high-throughput screening (HTS), 1, 171, 172 HINT, 2, 162

Hohenberg-Kohn (HK) theorem, 1, 11, 20

homodesmotic reactions, 1,34

homology models, 1, 170, 188, 189; 3, 211

HTD see high throughput docking

HTS data analysis, 2, 156

HTS Data Mining and Docking Competition, 2, 159

HTS see high-throughput screening

human intestinal oral plasma protein binding, 5, 103, 116

hybrid quantum and molecular mechanical simulation (QM/MM), 2, 263-268

hybrid solvent, 2, 106

hybridization, structure-based, 1, 191, 192

hydration free energies, 1, 103

Hylleraas Method, 2, 21

Hylleraas-CI method, 2 , 24

hyperdynamics, 2 , 221, 224, 225; $\underline{5}, 80,83-85$, $89,91-93$

IAPs, 2, 206

ICM, 2, 299, 300, 308, 313-314, 318-319

ICMRCI, 3, 163

IL-2, $2,21 \overline{4}$

implicit solvent, 2, 99-100; $\underline{3}, 5 ; \underline{4}, 107-109$, $111-113,117,125-134$

Induced Fit, 3, 218

information triple, 3, 109, 110, 128, 131

intermolecular potential functions, 1, 241, 242

internal coordinates, 3, 166

intestinal absorption, $\overline{1}, 137-138$

intestinal permeability, 1, 134, 135, 161

intrinsic errors, 3 , 196

iron chelation, modeling of, 2, 185

isodesmic/isogyric reactions, 1,34

Jacobi coordinates, 3, 158

Jarzynski relationshīp, 1, 103-110; $\underline{3}$, 45, 46

Jmol, 3 , 99, 113-117, 119-121, 125, 126

Kemp decarboxylation, 2, 263, 264, 271-273, 275

kinetics, $\underline{4}, 16,68,113,156,175,186,190-192$, 196

kinome targeting, 1, 185-202

applications, $\underline{1}, \overline{1} 92-197$

ATP site recognition, $1,187,188$

homology models, $1, \overline{1} 88,189$ kinase family, 1, 186, 187

methodology, $1,188-192$

selectivity, 1 , 190, 191

structure-based hybridization, 1, 191, 192

virtual screening, 1, 189, 190

knowledge-based scoring functions, $\underline{1}$, 123-125

knowledge bases, 4, 204, 208-214

Kohn-Sham (KS) equations, 1, 11, 20-22, 25

Kohonen maps, 2, 181

Kriging, 2, 151

laboratory course modules, $\underline{1}, 7$

Lamb-shift, $\underline{3}, 163,164$

Lambda dynamics, 3 , 6

Lanczos technique, $\overline{3}, 166$

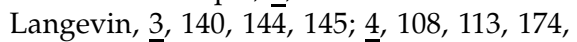
180,184

Landau-Zener theory, 5, 166

$\operatorname{LCCSD}(\mathrm{T}), 1,54,62,7 \overline{1}, 78$

$\operatorname{LCCSD(TO),}, \underline{1}, 64$

lead optimization see structure-based lead optimization

lead-likeness, 1, 159

Lennard-Jones (LJ) potential, 1 , 93, 94, 116, 121

LES see locally enhanced sampling

level density, $\underline{3}, 156$

library enumeration, 1,178

ligand binding, $\underline{1}, 10 \overline{3} ; \underline{3}, 42,43,51$

ligand-based screening, 1, 172-175, 178-9

LigandFit, 2, 299, 300, 302, 303, 315-17, 319

LigScore2, $\underline{2}, 161$

linear interaction energy, 1,117

Linear R12 methods, 2,28

linear scaling, 2, 54, 55, 62, 64, 77

LINGO, 2, 146

link atoms, 2, 37

LJ see Lennard-Jones

LMP2, 2, 55, 60-78

Local Correlation, 2, 53, 77

local coupled cluster, 2,54

local spin density approximation, $1,11-12$

localized orbitals, 2, 53, 54, 57

locally enhanced sampling (LES), $\underline{1}, 79$

LOOPSEARCH, $\underline{3}, 216$

LUDI scoring function, $\underline{1}, 123,173$

lysozyme, $\underline{2}, 199$

machine learning, 4, 4, 25, 41-46, 49, 53-58

many-body perturbation theory, 1,10

Maple, 1, 228, 230-232

MARVEL, $\underline{3}, 157-162,165$

master equations, $\underline{1}, 115,116,119,120$ 
Mathematical Association of America, 1, 215, 216

MaxFlux, $\underline{3}, 16$

maximum common substructure, 2, 160

maximum likelihood methods, $3, \overline{4} 4$

MC see Monte Carlo

MCSCF see multi-configurational self-consistent field

MCSS program, 1, 173, 174, 177

MD see molecular dynamics

MDM2, 2, 197, 200, 209-211

mechanical embedding, 2, 37

MEMBSTRUCK, 3, 220

membrane, $4,49,50,108,110,111,115-117$, $131 ; 5, \overline{4}-8,12,13,38,69,104,108,111$, $113, \overline{1} 15,116,119$

Menshutkin reaction, 2, 263, 265-268, 275

metabolic stability, $1, \overline{1} 42,143,162$

see also ADMET properties

metal surface, 3, 137

Miller indices $\bar{h}, k, l, \underline{3}, 91$

MLR, $\underline{3}, 67$

MLR see multiple linear regression

MM see molecular mechanics

model applicability domain, $\underline{3}, 68,74$

Model scope, 2, 155

MODELLER, $\overline{3}, 213$

MODLOOP, 3, 216

MOE, 3, 214

MOEDock, 2, 299, 300, 317

MOIL, 3,19

molecular crowding, $\underline{4}, 110$

molecular descriptors, $\underline{2}, 141,144-146,151$; $\underline{3}$, 66

molecular dynamics, 2, 98, 99, 221-224, 227-230, 233-238, 243, 244, 246, 247; 3, $140 ; 4,33,72,109,111,112,117,126, \overline{13} 3$, $134, \overline{1} 39,146,147,161-163$

atomistic models, 3, 143

coarse-grained, $3, \overline{1} 38,144$

with electronic friction, $\underline{3}, 143$

molecular dynamics (MD) simulation, 1 , 75-78, 217, 239, 242

molecular interaction field, $\underline{3}, 66$

molecular mechanics (MM), 1, 119-122

molecular modeling, $1,59-1 \overline{30}$

atomistic simulation of nucleic acids, 1 , 75-89

free energy, $1,103-111,113-130$

nonequilibrium approaches, $1,103-111$

protein force fields, $\underline{1}, 91-102$

protein-ligand interactions, 1 , 113-130

water models, 1 , 59-74

TIP4P, $1,62-64,69-72$

TIP4P-EW, 1 , 64, 65, 69-72
TIP5P, 1, 65-67, 69-72

TIP5P-E, 1, 67-72

molecular orbital representation, 1 , 229-231

Molecular Similarity, 2, 141

molecular simulations, $\underline{1}$, 177, 178, 239-244; $\underline{4}$, 134

Møller-Plesset form, 1, 10, 48-50

MOLPRINT 2D, 2, $14 \overline{5}$

Monte Carlo methods, 1 , 216-218, 239, 242, 247, 248

Monte Carlo simulation (MC), 2, 263-268, $270,271,273,275 ; 5,49,70$

multi-configurational self-consistent field (MCSCF) method, 1, 9, 10, 46, 47

multicanonical ensemble, 5, 69

multicanonical methods, $\underline{3}, 48$

MULTIMODE, 3, 166

multiple excitations, 1,25

multiple linear regression (MLR), 1, 136

multiple sequence alignment, 3 , 2 $\overline{1} 1-213$

multipole approximations, $2, \overline{62}$

multireference methods, $1, \overline{5} 1-53$

MV, 3, 163

MVD1, 3, 164

MVD2, $\underline{3}, 163$

n-mode representation, $\underline{3}, 167$

$\mathrm{N}_{2} \mathrm{O}, \underline{3}, 162$

$\mathrm{N} 1.50,3,211$

N7.49, $\overline{3}, 211,212$

National Science Foundation (NSF), 1, 206, 207, 209

neural networks, 2, 181

nonadiabatic, $\underline{3}, 1 \overline{5} 8$

nonequilibrium approaches

computational uses, 1,109

experimental applications, 1, 108

free energy calculations, $1, \overline{1} 03-111$

Jarzynski relationship, $1, \overline{1}$ 103-110

theoretical developments, 1, 108, 109

NMR, 4 $, 10,29,31,53,68,75,82,90-92$, 96-102, 139-141, 143-147, 149, 151, 152, 162,206

nonequilibrium work, $\underline{3}, 45,46$

nonlinear models, $2,1 \overline{52}$

normal coordinates, $\underline{3}, 163,167,168$

normal mode, 3,159

NPXXY motif, $\underline{\underline{3}}, 212$

NR, 2, 211

NSF see National Science Foundation

nuclear hormone receptor, 2, 211

nuclear motion computations, $\underline{3}, 166$

nuclear-motion, 3, 169

nucleic acids, $1,75-89$ 
nucleophilic aromatic substitution $\left(\mathrm{S}_{\mathrm{N}} \mathrm{Ar}\right), \underline{2}$, 263,264

nudged-elastic-band (NEB) method, 3,16

nuisance compounds, $\underline{1}, 162,163,190$

objectives for teaching crystallography, $\underline{3}$, 86-89

OMTKY3, 3, 189

ONIOM, 2, 35

Onsager-M̄achlup action, 3, 17, 18

OPLS-VA/VA force fields, $2,265,273$

OPLS/AA force fields, $1,92-94,97$

optical interference, $3, \overline{96}$

oral bioavailability, 1, 134, 138, 139, 159, 160

oral drug activity, $1,159,160$

orbital domains, $\underset{2}{2}$,58, 59, 61-63

orbital representations, $1,225-231$

orthogonal coordinates, $\underline{3}, 166$

oscillating systems, $\underline{1}, 2 \overline{2}, 233$

overfitting, 2, 154

p-glycoprotein, 1 , 140, 160-161

p53, 2, 197, 200, 209-211

PAO, 2, 53-62, 68

parallel computing, 1,242

parallel-replica dynamics, $\underline{5}, 81,83,88,90,96$

PARAM force fields, 1,97

partial least squares (PLS), 3, 67

partial least squares (PLS) analysis, 1 , 134, 135, 138

patterning, 1, 247

PB see Poisson-Boltzmann

PCM, 2, 266, 271, 275

PCM induced charges, $\underline{3}, 181$

PDB see Protein Data Bank

PDBbind, 2, 161

PDDG/PM3 , 2, 263-265, 267, 268, 273-275

PDF inhibitor, 2, 288

periodic boundary conditions, $\underline{3}, 181$

permeability, intestinal, 1, 134, 135, 161

perturbation theory (PT), $1,10,51,52$;

$$
\underline{3}, 156
$$

PES see potential energy surface

pH-coupled molecular dynamics, $\underline{3}, 4$

$\mathrm{pH}$-modulated helix-coil transitions, $\underline{3}, 9$

pharmaceutical chemicals

ADMET properties, 1, 133-151

drug discovery, $1,1 \overline{5}-168$

structure-based lead optimization, $\underline{1}$,

169-183

virtual screening protocols, 1 , 114, 120, 125

pharmacophore models, $1,172-174$

pharmacophores, 2, 182, 183

PhDOCK, 1 , 173, 174, 177 phospholipid, $\underline{5}, 6,11,16$

physical chemistry, 1 , 215-217

Pipek-Mezey localization, 2, 56, 68

$\mathrm{p} K_{\mathrm{a}}, 3,4,188$

$\mathrm{p} K_{\mathrm{a}}$ prediction, 3,4

$\mathrm{p} K_{\mathrm{a}}$ values, $4,7 \overline{3}, 90-94,96-100,102$

plasma protein binding (PPB), 1,142

PLOP, 3 , 216

PLP2, 2, 161

PLS see partial least squares

PMF, 2, 161, 162, 263, 266

PMFScore, 1, 124, 125

Podcast, 3 , 99 , 118-121, 131

point group symmetry, 3,94

Poisson-Boltzmann (PB) equation, 1 , 117-122; 4, 97, 109, 129

polarizable continuum model (PCM), 2, 264, 266,271

polarization consistent, $\underline{3}, 196$

polymerization, $\underline{4}$, 174, $175,177,179-192$, 194-196

polymer-source chemical vapor deposition (PS-CVD), 1, 232, 233

polynucleotides, $\underline{5}, 59,65$

poly(organo)silanes, $1,232,233$

polypeptides, $\underline{5}, 59, \overline{61}, 65,69,164-166$, $168-170,172,173,175,176$, 180,181

pores, $\underline{5}, 6,12,14-16$

positive desirable chemistry filters, $\underline{1}, 158,159$

PostDOCK, 2, 157

potential energy landscape, 2, 221-224, 227, 229,230

potential energy surface (PES), 1, 3, 4, 54

potential functions, $\underline{1}, 241,242$

potential of mean force (PMF), 2, 263-268

PPB see plasma protein binding

PREDICT, 3, 219

predictive modeling, 1 , 133-151, 240

PRIME, 3, 214

principal component, 5, 39-41, 61, 120

principal component analysis, 2, 233, 235,236

privileged structures, 1,158

probabilistic protein design, 1, 249, 250

problem-solving templates, $\underline{1}, 228$

process design, 1, 231, 232

projected atomic orbitals, 2, 53

projective models, $\underline{3}, 144$

proline, 3 , 213, 216, 221

promiscuous compounds, $\underline{1}, 162,163,190$

protein $\mathrm{A}, \underline{3}, 22$

protein conformational change, $\underline{4}, 101,161$, 162

Protein Data Bank (PDB), 1, 113, 117, 123, 124 
protein design, 1 , 245-253

degrees of freedom, 1, 246

energy function, $1,2 \overline{4} 6,247$

examples, 1, 248-250

search methods, 1 , 247, 248

solvation and patterning, 1,247

target structures, $\underline{1}, 246$

protein electrostatics, $\underline{4}, 90,102$

protein folding, 3 , 22

protein force fields, $1,91-102$

condensed-phase, $\underline{1}$, 94-96

free energies of aqueous solvation, 1,96

gas-phase, 1 , 94-96

optimization, 1, 96-99

united-atom, $\overline{1}, 97$

protein function, $4,5-7,49,67$

protein kinases see kinome targeting

protein misfolding and aggregration, $\underline{3}, 9$

protein-ligand interactions, $1,113-130$

protein-protein interaction, $\overline{2}$, 197-198, 200, 202, 203, 205, 211, 214, 215

protein structure, 4 , 4-6, 9, 10, 13-15, 17, 24, $30,42,49,50,53,54,56,58,90,91,93$, 96-102, 112, 208

protein-RNA, 4, 49

PS-CVD see polymer-source chemical vapor deposition

pseudopotentials, $\underline{3}, 200$

PubChem, 4, 204, 205, 211-213, 218-227, 229-240

QED, 3, 158, 163

$\mathrm{QM} / \mathrm{EFP} / \mathrm{PCM}, 3,181$

QM/MM, 2, 35, 263-268, 270, 271, 273-275; 3 , $182,188,190 ; 4,156-164$

QSAR, 3, 66; 푸 104, 105, 107, 109, 110, $115-118, \overline{1} 20-122$

QSAR/QSPR models, 1, 133-151

quantum electrodynamics (QED), $\underline{3}, 155$

quantum mechanics, $1,3-56$

basis sets, $1,13-15,32,33$

bond breaking, 1, 45-56

computational thermochemistry, $1,31-43$

configurational interaction, $\underline{1}, 9, \overline{10}, 48,51$

coupled cluster methods, $1,10,11,37-40$, $48-50,52,53$

density functional theory, $1,4,11,12$,

$13-15,32,33,48,49$

electron correlation methods, $1,8-11$

generalized valence bond method, $1,47,48$

Hartree-Fock method, 1, 4, 5-11, 13-15, 20,

$21,46,48-51$

perturbation theory, $1,10,51,52$

potential energy surface, $1,3,4,54$ self-consistent field methods, 1, 6-10, 37, $46,47,53$

semi-empirical methods, $1,12-13,15$

symbolic computation engines, 1, 225-228

time-dependent density functional theory, $1,20-30$

quantum number, 3,164

quantum-classical enzymatic calculations, $\underline{1}$, 103

quasi-static (QS) transformations, 1, 105, 133-151

QZVPP, 3, 197

R-group descriptor, 2, 147

random Forest, $2,13 \overline{6}, 151$

rare event, $\underline{3}, 14 \overline{0}$

RASSCF see restricted-active-space selfconsistent field

re-parameterizations, 1 , 59-61, 67, 72

reaction energies, 2 , $5 \overline{3}, 54,64,71,74,75,77$

reaction kinetics, $\underline{3}, 158$

receptor activation, 3,221

refinement, 3 , 216, 21 18,219

relativity, $\underline{3}, \overline{2} 00$

REMD see Replica Exchange Molecular Dynamics

Replica Exchange Molecular Dynamics, 2, 83, 85, 87, 89-91, 93, 95, 222

Replica exchange with solute tempering (REST), 2, 86

replica-exchange, $\underline{3}, 7$

repository, $\underline{4}, 10,5 \overline{6}, 205,218,238$

Research Experiences for Undergraduates (REU), 1, 209

research institutions, $\underline{1}, 205-214$

restrained electrostatic potential, $1,92,93$

restricted Hartree-Fock (RHF), 1, 46, 48-50

restricted-active-space self-consistent field (RASSCF) method, 1, 47

REU see Research Experiences for Undergraduates

RHF see restricted Hartree-Fock

RISM, 2, 266, 267

ROC curve, 2, 297, 306, 307, 315

ROCS, 2,318

Roothaan-Hall equations, $1,6-8$

rotational-vibrational

energy levels, 3 , 159

spectra, $\underline{3}, 169$

transitions, $\underline{3}, 159$

rovibrational eigenvalues, $\underline{3}, 157$

$\mathrm{Ru}(\mathrm{bpy})_{3}^{2+} 7$

Runge-Gross theorem, 1, 27

Rydberg orbital, $\underline{5}$, 165-168, 170-178 
$\mathrm{S}_{\mathrm{N}} \mathrm{A}, 2,270,271$

$\mathrm{S}_{\mathrm{N}} \mathrm{Ar}, 2,268-270,275$

sampling barriers, 1, 242, 243

SAR see structure-activity relationships scads, 1,250

scaling methods, 1, 6-8

Schrödinger equation, 1, 3-15; 2, 297-299, $313,314,316,318-320$

scoring functions, $1,119-126$

scoring functions, quality, 2, 161, 162

self-consistent field (SCF) methods, 1, 6-10, $37,46,47,53$

self-consistent reaction field (SCRF), 1, 118, 121

self-extracting databases, 1, 223, 225

self-learning hyperdynamics, 5 , 89, 92, 93

selectivity, 4, 23-27, 29, 33, 74

semantic Wiki, 3 , 110, 123, 126-128, 131

semi-empirical methods, $1,12-13,15,31,32$

PDDG/PM3, 2, 264, 265, 267, 268, 272, 274, 276

sextic force fields, 3,162

SHAKE algorithm, 2,222

signal trafficking see kinome targeting

similar property principle, 2,141

simulation, $4,9,33,72,74, \overline{7} 7,78,81,82$, 107-109, 111-115, 117, 126, 128-134, 139-144, 146-152, 156, 159-164, 184, 187-192, 194, 195

Slater geminal methods, 2 , 28, 30

Smac, 2, 206, 208, 209

small molecule solvation, $\underline{3}, 50$

"soft core" Lennard-Jones interactions, 3, 47 solubility, 1 , 135-7; 5 , 104-107, 111, 113, 114 , $119,1 \overline{2} 2,123$

solvation, 1 , 117-119, 247

space group symmetry, 3,94

spectroscopic accuracy, $\overline{3}, 157$

spectroscopic network ( $\overline{\mathrm{S}} \mathrm{N}), \underline{3}, 159$

spherical harmonics, 3, 167

spin-flip methods, $1, \overline{5} 3$

spin relaxation, $\underline{4}, \overline{139}, 140$

standard domains, 2 2, 53, 57, 59, 64, 68, 69, 71, 73-76

standard $\mathrm{p} K_{\mathrm{a}}, \underline{3}, 4$

standard uncertainty (su), $\underline{3}, 87$

statistical computational assisted design strategy (scads), 1, 250

Steepest Descent Path (SDP), $\underline{3}, 19$

stochastic difference equation in length (SDEL), 3, 17-19

advantages, $\underline{3}, 20$

disadvantages, $\underline{3}, 20$

stochastic difference equation in time (SDET), 3,17
Stochastic Gradient Boosting, 2, 137

stochastic models, 1 , 215-220

storage capacity, $1,224,225$

string method, 3,16

strong pairs, $2, \overline{5} 9,62,63,68-9,71,73,75,77$

structural mimicry, $\underline{3}, 217$

structural motifs, 3,211

structure-activity, $\overline{4}, 24,27,47,159,208,227$, 232-235

structure-activity relationships (SAR), 1, 91, 133-151; 4, 24, 159, 161, 204, 208, 210-212, 232

Structure-based design, 2, 197, 202, 205, 209

structure-based drug design, $1,114,120,125$; $4,33,160$

structure-based hybridization, 1, 191, 192

structure-based lead optimization, 1, 169-183

application to specific targets, 1,179

compound equity, 1,171

discovery, $1,171-1 \overline{7} 5$

fragment positioning, 1, 175-177

high-throughput screening, 1, 171, 172

library enumeration, 1,178

ligand-target complex evaluation, $\underline{1}, 178$, 179

modification, 1 , 175-179

molecular simulation, $\underline{1}, 177,178$

structure visualization, 1,175

virtual screening, $1,169,172-175$

structure-based ligand design, 2, 184

structure-based virtual screening, 2, 284

structure-property relationships, 2, 142

structured-prediction, $\underline{4}, 44,48-5 \overline{0}, 53-55,57$

substrate access, $\mathrm{P} 450, \overline{2}, 178$

substrate prediction, $\mathrm{P} \overline{450}, 2,172$

support vector machines, $\underline{1}, 137,145 ; \underline{2}, 128$, 149

surface diffusion, $3,138,140$

Surflex, 2, 161

Sutcliffe-Tennyson triatomic rovibrational Hamiltonian, $\underline{3}, 167$

symbolic computation engines (SCE), $\underline{1}$, 221-235

advanced application-specific procedures, $1,229-231$

computation power, 1 , 228, 229

emulation of professional software, $\underline{1}$, 229-231

graphical representations, 1 , 225-228, 232, 233

process design, 1 , 231, 232

quantification, 1, 225, 231-233

self-extracting databases, 1,223

specialized procedures, $1,228,229$

storage capacity, $\underline{1}, 224, \overline{2} 25$ 
T4 lysozyme, 3, 52

target structures, $\underline{1}, 246$

TASSER, $\underline{3}, 220$

tautomeric interconversion, $\underline{3}, 7$

TC5b, 2, 89

TDDFT see time-dependent density functional theory

temperature accelerated dynamics, $\underline{5}$, 81, 85, 86

temperature programmed-desorption, 2, 6

template approach, 1, 228, 229

thermal conductivity, 1, 242, 243

thermochemistry, $\underline{3}, 158$

thermochemistry, computational, 1, 31-43

thermodynamic integration (TI), $\overline{3}, 4445$

thermodynamics

integration method, 1, 104

nonequilibrium approaches, 1, 103-111

protein-ligand interactions, 1, 113-130

symbolic computation engines, 1 , 224, 225

water models, 1 , 59-72

thermogravimetric analysis, 2, 6

thermostat, 4 , 113, 148

thyroid hormone, $\underline{2}$, 197, 201, 211

time-dependent density functional theory

(TDDFT), 1, 20-30

computational aspects, $1,21,22$

developments, 1 , 26-28

electronic excitations, 1, 20, 21

exact exchange, $1,26,27$

performance, $1, \overline{2} 2-24$

qualitative limitations, $1,25,26$

time-dependent Hamiltonian operators, $\underline{1}$, 104

time-independent Schrödinger equation, $\underline{3}$, 167

TIP3P, 2, 86, 89, 266

TIP4P, 1 , 62-64, 69-72; 2, 265-267

TIP4P-Ew, 1, 64-65, 69-72

TIP5P, 1, 65-67, 69-72

TIP5P-E, $1,67-72$

titration curves, 4, 90-94, 96-99, 101, 102

TKL see tyrosine kinase-like

TKs see tyrosine kinases

toggle switch, 3, 212

Top7, 1, 249

torsional space, $5,27,52,53$

toxicity, $1,144,190$

see also ADMET properties

TR, 2, 212

transamination, $1,232,233$

transferable intermolecular potential (TIP)

water molecules, 1, 59-74

transient complex, $4,7 \overline{5}, 77-81$

transition path sampling (TPS), $\underline{3}, 16$ transition path theory, 3, 16

transition state theory, $\underline{2}, 224,229 ; \underline{3}, 141$

Trp-cage, 2, 89, 90, 93

Turbo Similarity Searching, 2, 153

two-electron integrals, 1, 6-7, 12, 13; 3, 182

tyrosine kinase-like (TKL) group of kinases, 1, 186, 196-197

tyrosine kinases (TKs), 1, 186, 194, 195

UHF see unrestricted Hartree-Fock

umbrella potential, 2, 223

umbrella sampling, $\underline{2}, 221,223,224$, 228,230

undergraduate research, 1, 205-214

Undergraduate Research Programs (URPs), $1,208-212$

united-atom protein force fields, 1,97

university research, 1 , 205-214

unrestricted Hartree-Fock (UHF), 1, 46, 50,51

URPs see Undergraduate Research Programs

van't Hoff reactions, 1, 228, 229

vertical excitation, $\underline{1}, \overline{2} 2-24$

vibrational

band origins (VBOs), $\underline{3}, 164,168$

energy levels, $\underline{3}, 161$

states, $\underline{3}, 160$

virtual database screening, 2, 201

virtual screening, $\underline{1}$, 169, 172-175, 189, 190; 2 , 158

high throughput, 1,120

protocols, $\underline{1}, 114, \overline{120}, 125$

Virtual Screening, performance assessment of algorithms, 2, 144

viscosity, $1,242, \overline{2} 43$

visualization, 1 , 175, 225-228, 232, 233

VPT2, $\underline{3}, 163$

water dimer, 3 , 188

water models, 1 , 59-74; 2, 98, 102

bio-molecular simulation, 1, 59-61

effective fragment potential (EFP), 2, 267

five-site, $1,65-72$

four-site, 1, 62-65, 69-72

generalized conductor-like screening

model (GCOSMO), 2, 266

methods, 1, 61, 62

reference interaction site model (RISM), 2 , 267, 268

TIP3P, 2, 266, 267

TIP4P, 1 , 62-64, 69-72; 2, 265-267

TIP4P-Ew, 1, 64, 65, 69-72 
TIP5P, 1, 65-67, 69-72

TIP5P-E, $1,67-72$

water-benzene dimer, $3,186,188$

wavefunctions, $1,225-\overline{2} 28$

weak pairs, 2, $6 \overline{2}-63,68$

Web 2.0, 3, 100, 111, 122, 124, 131

web-based tools, 4, 237

Weighted Probe Interaction Energy Method, 2, 147

Weizmann- $n$ theory, 1 , 37-39

Wigner rotation functions, 3,166

Wiki, 3, 99, 103, 108, 117, 121-131
Wikipedia, 3, 99, 112, 122, 124, $129,13 \overline{1}$

Wn (Weizmann-n), $\underline{3}, 160$

XED, 2, 159

XIAP, 2, 206, 208, 209

XScore, 1 , 123; 2, 161, 162

Z-factor equation, 1 , 22

zeolites, 2, 45

Zwanzig relationship, 3 , 43, 44 